\title{
STRATIFIED PATH SPACES AND FIBRATIONS
}

\author{
Bruce Hughes
}

\begin{abstract}
The main objects of study are the homotopically stratified metric spaces introduced by Quinn. Closed unions of strata are shown to be stratified forward tame. Stratified fibrations between spaces with stratifications are introduced. Paths which lie in a single stratum except possibly at their initial points form a space with a natural stratification, and the evaluation map from that space of paths is shown to be a stratified fibration. Applications to mapping cylinders and to the geometry of manifold stratified spaces are expected in future papers.
\end{abstract}

\section{INTRODUCTION}

Spaces with stratifications are decomposed into disjoint subspaces called strata. Two strata are adjacent if one of them (the lower stratum) is contained in the closure of the other. Quinn [14] defines homotopically stratified spaces in terms of homotopy theoretical properties of pairs of adjacent strata, the defining conditions essentially implying that there is a good homotopy theoretical model for a normal fibration of one strata in the other. In addition to conditions on pairs of adjacent strata, it is desirable to have an understanding of the nature of the embedding of a stratum (or a closed union of strata) in the entire space. For example, instead of just knowing that the local homotopy type of a pair of adjacent strata is locally constant along the lower stratum, one would like to know that the stratified local homotopy of the space is locally constant along any stratum. The main object of this paper is to develop such a global understanding.

The paper [6] announces a generalized Tubular Neighborhood Theorem for homotopically stratified spaces with manifold strata. For spaces with only two strata a complete proof was given in [9]. The present paper is the first in a series (culminating in [7]) which will provide a proof of the general case.

To motivate the main results, consider a locally finite simplicial complex $X$. Such a space is an example of the type of stratified space of interest here: the strata are the open simplices. If $Y$ is a subcomplex of $X$, then $Y$ is a closed union of strata. The classical Homotopy Extension Property implies that any deformation $f: Y \times I \rightarrow Y$ (i.e., a self-homotopy with $h_{0}=\mathrm{id}_{Y}$ ) extends to a deformation $\tilde{f}: X \times I \rightarrow X$. In general, the extension $\tilde{f}$ cannot be required to preserve the complement of $Y$ (i.e., one cannot require $\tilde{f}((X \backslash Y) \times I) \subset X \backslash Y)$. This is because the local homotopy type of the complement might not be locally constant along $Y$.

1991 Mathematics Subject Classification. Primary 55R55, 55R65; Secondary 57N80, 58A35.

Key words and phrases. stratified space, strata, homotopy link, stratified fibration, stratified approximate fibration, stratified forward tame.

Supported in part by NSF Grant DMS-9504759 
(For example, if $X$ is the union of three 1-simplices with a single common vertex $v$ and $Y$ is the union of two of the 1-simplices, then a deformation of $Y$ which moves $v$ cannot be extended to a complement preserving homotopy of $X$.) However, if $f$ is a stratum preserving deformation (i.e., for each $y \in Y, f(\{y\} \times I)$ lies in the same open simplex of $Y$ as $y$ ), then the extension $\tilde{f}$ can be required to preserve the complement of $Y$; in fact, $\tilde{f}$ can be required to be stratum preserving.

This stratum preserving deformation extension property is easily verified for simplicial complexes by extending the deformation over one simplex at a time. As an application of our main results we will verify the extension property for a homotopically stratified metric space (with finitely many strata) with $Y \subseteq X$ a closed union of strata. The crux of the problem is to extend the stratum preserving deformation $f: Y \times I \rightarrow Y$ to a neighborhood $U$ of $Y$ in $X$. The first step for this (and the first part of the main theorem below) is to prove that $Y$ is stratified forward tame in $X$ : there exists a neighborhood $U$ for which there is a deformation $h: U \times I \rightarrow X$ of $U$ to $Y$ in $X$ rel $Y$ which is nearly stratum preserving in the sense that $h$ is stratum preserving except at time $t=1$ when $h_{1}(U)=Y$.

The second step is to notice that the deformation $f$ and the nearly stratum preserving deformation $h$ combine to give a homotopy lifting problem into the space $\mathrm{P}_{\text {nsp }}(X, Y)$ of nearly stratum preserving paths in $X$ with end point in $Y$. $\mathrm{P}_{\text {nsp }}(X, Y)$ maps to $Y$ by evaluation. A stratum preserving solution solves the extension problem (for more details see $\S 6$ ). Such a solution exists by the second part of the main theorem.

We now state the main theorem and the corollary regarding stratum preserving deformation extension, referring to the body of the paper for more complete definitions. The two parts in the theorem are global versions of the Forward Tameness and Normal Fibrations conditions in Definition 3.3 below.

Main Theorem. Let $X$ be a homotopically stratified metric space with a finite number of strata and let $Y \subseteq X$ be a closed union of some of the strata of $X$. Then

(1) $Y$ is stratified forward tame in $X$, and

(2) the evaluation map $q: \mathrm{P}_{\mathrm{nsp}}(X, Y) \rightarrow Y$ is a stratified fibration.

Corollary (Stratum Preserving Deformation Extension Property). Let $X$ be a homotopically stratified metric space with a finite number of strata and let $Y \subseteq X$ be a closed union of some of the strata of $X$. If $f: Y \times I \rightarrow Y$ is a stratum preserving deformation, then there exists a stratum preserving deformation $\tilde{f}: X \times I \rightarrow X$ extending $f$.

One purpose of this paper is to provide some foundational material on stratified fibrations and stratified approximate fibrations. Quinn [13] has previously considered stratified systems of fibrations in which the range of a map is stratified and over each stratum there is an ordinary fibration (with a mild compatibility condition). For stratified fibrations, both domain and range are stratified and the homotopy lifting problem and solution are required to respect these stratifications.

Here is one way the homotopy theoretical results of this paper will be used. Limits of certain stratum preserving geometric constructions will be taken in [7]. The stratum preserving property will be lost in the limit because of collapsing of strata phenomena. The fact that $q: \mathrm{P}_{\mathrm{nsp}}(X, Y) \rightarrow Y$ is a stratified fibration will be used to lift the collapse and recover stratum preservation. 
In addition, we plan to use the results of this paper to verify that mapping cylinders of certain maps between manifold stratified spaces are themselves manifold stratified spaces. This line of research is related to the work of Cappell and Shaneson [3].

These results are closely related to those of Quinn [14, 3.2]. However, the proofs given here are independent of [14], and correct certain technical deficiencies in [14]. For more information see $\S 10$.

\section{BACKGROUND ON SPACES WITH STRATIFICATIONS}

This section contains the basic definitions from the theory of stratifications together with a few observations which are well-known to the experts. For other treatments of this foundational material, consult Akbulut and King [1], Dovermann and Schultz [4], Goresky and MacPherson [5], Verona [17], and Mather [11],[12].

Definition 2.1. A partition of a space $X$ consists of an index set $\mathcal{I}$ and a collection $\left\{X_{i}\right\}_{i \in \mathcal{I}}$ of pairwise disjoint subspaces of $X$ such that $X=\cup_{i \in \mathcal{I}} X_{i}$. For $i \in \mathcal{I}, X_{i}$ is called the $i$-stratum.

Definition 2.2. A stratification of a space $X$ consists of an index set $\mathcal{I}$ and a locally finite partition $\left\{X_{i}\right\}_{i \in \mathcal{I}}$ of locally closed subspaces of $X$. For $i \in \mathcal{I}, X_{i}$ is called the $i$-stratum and

$$
X^{i}=\cup\left\{X_{k} \mid X_{k} \cap \operatorname{cl}\left(X_{i}\right) \neq \emptyset\right\}
$$

is called the $i-$ skeleton. In this case, $X$ is a space with a stratification.

Note that the skeleta are closed subspaces of $X$. For if $x \in X \backslash X^{i}$, then $x \in X_{k}$ for some $k \neq i$ and $X_{k} \cap \operatorname{cl}\left(X_{i}\right)=\emptyset$ so $x \notin \operatorname{cl}\left(X^{i}\right)$.

For a space $X$ with a stratification $\left\{X_{i}\right\}_{i \in \mathcal{I}}$, define a relation $\leq$ on the index set $\mathcal{I}$ by

$$
i \leq j \quad \text { if and only if } \quad X_{i} \subseteq \operatorname{cl}\left(X_{j}\right) .
$$

The stratification satisfies the Frontier Condition if for every $i, j \in \mathcal{I}$,

$$
X_{i} \cap \operatorname{cl}\left(X_{j}\right) \neq \emptyset \quad \text { implies } \quad X_{i} \subseteq \operatorname{cl}\left(X_{j}\right) .
$$

Proposition 2.3. If a stratification $\left\{X_{i}\right\}_{i \in \mathcal{I}}$ of $X$ satisfies the Frontier Condition, then

(1) $\leq$ is a partial ordering of $\mathcal{I}$,

(2) for every $i, j \in \mathcal{I}, X^{i} \subseteq X^{j}$ if and only if $i \leq j$,

(3) for each $i \in \mathcal{I}, X^{i}=\operatorname{cl}\left(X_{i}\right)$.

Proof. (1) The reflexive and transitive properties are clear. To establish antisymmetry, assume $X_{i} \subseteq \operatorname{cl}\left(X_{j}\right)$ and $X_{j} \subseteq \operatorname{cl}\left(X_{i}\right)$ and show that $X_{i}=X_{j}$. Since $X_{i}$ is locally closed, given $x \in X_{i}$ there exists an open neighborhood $U$ of $x$ in $X$ such that $U \cap X_{i}$ is closed in $U$. Note that $U \cap X_{i}=U \cap \operatorname{cl}\left(X_{i}\right)$. Since $x \in \operatorname{cl}\left(X_{j}\right)$ there exists $y \in U \cap X_{j} \subseteq U \cap \operatorname{cl}\left(X_{i}\right)=U \cap X_{i}$. Thus $X_{i} \cap X_{j} \neq \emptyset$ and so $X_{i}=X_{j}$.

(2) Suppose first that $X^{i} \subseteq X^{j}$. Since $X_{i} \subseteq X^{i} \subseteq X^{j}$, it follows that $X_{i} \cap$ $\operatorname{cl}\left(X_{j}\right) \neq \emptyset$. The Frontier Condition implies that $X_{i} \subseteq \operatorname{cl}\left(X_{j}\right)$ so $i \leq j$. Conversely, suppose $i \leq j$ so that $X_{i} \subseteq \operatorname{cl}\left(X_{j}\right)$. If $X_{k} \cap \operatorname{cl}\left(X_{i}\right) \neq \emptyset$, then $X_{k} \cap \operatorname{cl}\left(X_{j}\right) \neq \emptyset$, so $X^{i} \subseteq X^{j}$.

(3) Since $X_{i} \subseteq X^{i}$ and skeleta are closed, $\operatorname{cl}\left(X_{i}\right) \subseteq X^{i}$. If $X_{k} \cap \operatorname{cl}\left(X_{i}\right) \neq \emptyset$, then the Frontier Condition implies that $X_{k} \subseteq \operatorname{cl}\left(X_{i}\right)$, so $X^{i} \subseteq \operatorname{cl}\left(X_{i}\right)$. 
Corollary 2.4. If $\left\{X_{i}\right\}_{i \in \mathcal{I}}$ is a stratification of $X$, then the Frontier Condition holds if and only if $\leq$ is a partial ordering of $\mathcal{I}$ and for each $i \in \mathcal{I}, X^{i}=\operatorname{cl}\left(X_{i}\right)$.

Proof. If the Frontier Condition holds, use Proposition 2.3. Conversely, to verify the Frontier Condition assuming the sufficient conditions, assume that $X_{i} \cap \operatorname{cl}\left(X_{j}\right) \neq \emptyset$. Since $X^{j}=\operatorname{cl}\left(X_{j}\right)$ by assumption and $X_{i} \subseteq X^{j}$, it follows that $X_{i} \subseteq \operatorname{cl}\left(X_{j}\right)$.

Remark. In the terminology of Goresky and MacPherson [5, p.36] a stratification $\left\{X_{i}\right\}_{i \in \mathcal{I}}$ of a space $X$ satisfying the Frontier Condition is an $\mathcal{I}$-decomposition of $X$ and the strata $X_{i}$ are called pieces.

Definition 2.5. A filtration of a space $X$ consists of a partially ordered index set $(\mathcal{I}, \leq)$ and a collection $\left\{X^{i}\right\}_{i \in \mathcal{I}}$ of subspaces of $X$ such that for every $i, j \in \mathcal{I}$, $X^{i} \subseteq X^{j}$ if and only if $i \leq j$. For $i \in \mathcal{I}, X^{i}$ is called the $i$-skeleton and

$$
X_{i}=X^{i} \backslash \bigcup\left\{X^{j} \mid j<i\right\}
$$

is called the $i$-stratum. In this case, $X$ is a filtered space.

Note that a minimal element $-\infty$ and a maximal element $\infty$ may be adjoined to $\mathcal{I}$ so that $X^{-\infty}=\emptyset$ and $X^{\infty}=X$.

If $X$ has a filtration, then it is often the case that the associated strata define a stratification of $X$. For example, this happens if the skeleta in the filtration are closed in $X$, the strata are pairwise disjoint and the index set is finite. Conversely, it follows from Proposition 2.3 above that the skeleta induced by a stratification satisfying the Frontier Condition forms a filtration.

\section{QUINN'S THEORY OF STRATIFIED SPACES}

Some definitions from Quinn [14] are recalled (see also [6], [8], [9]).

Definition 3.1. A subset $Y \subseteq X$ is forward tame in $X$ if there exist a neighborhood $U$ of $Y$ in $X$ and a homotopy $h: U \times I \rightarrow X$ such that $h_{0}=$ inclusion $: U \rightarrow X$, $h_{t} \mid Y=$ inclusion $: Y \rightarrow X$ for each $t \in I, h_{1}(U)=Y$, and $h((U \backslash Y) \times[0,1)) \subseteq X \backslash Y$.

Definition 3.2. For $Y \subseteq X$ the homotopy link of $Y$ in $X$ by

$$
\operatorname{holink}(X, Y)=\left\{\omega \in X^{I} \mid \omega(t) \in Y \text { if and only if } t=0\right\} .
$$

Evaluation at 0 defines a map $q: \operatorname{holink}(X, Y) \rightarrow Y$ called holink evaluation.

Definition 3.3. A space $X$ with a stratification satisfying the Frontier Condition is a homotopically stratified space if the following two conditions are satisfied:

(i) Forward Tameness. For each $k>i$, the stratum $X_{i}$ is forward tame in $X_{i} \cup X_{k}$.

(ii) Normal Fibrations. For each $k>i$, the holink evaluation

$$
q: \operatorname{holink}\left(X_{i} \cup X_{k}, X_{i}\right) \rightarrow X_{i}
$$

is a fibration.

If $X$ is a space with a partition, then a map $f: Z \times A \rightarrow X$ is stratum preserving along $A$ if for each $z \in Z, f(\{z\} \times A)$ lies in a single stratum of $X$. In particular, a map $f: Z \times I \rightarrow X$ is a stratum preserving homotopy if $f$ is stratum preserving along $I$. 
Definition 3.4. A subset $Y \subseteq X$ of a space with a stratification is stratified forward tame in $X$ if there exist a neighborhood $U$ of $Y$ in $X$ and a homotopy $h: U \times I \rightarrow X$ such that $h_{0}=$ inclusion $: U \rightarrow X, h_{t} \mid Y=$ inclusion $: Y \rightarrow X$ for each $t \in I, h_{1}(U)=Y, h((U \backslash Y) \times[0,1)) \subseteq X \backslash Y$, and $h$ is stratum preserving along $[0,1)$.

Note that the homotopy $h$ need not be stratum preserving, but it is nearly stratum preserving.

\section{Stratified Path SPACES}

Let $X$ be a space with a stratification $\left\{X_{i}\right\}_{i \in \mathcal{I}}$ satisfying the Frontier Condition so that $\leq$ is a partial order on $\mathcal{I}$. All spaces of paths are given the compact-open topology.

If $Y \subseteq X$, then the stratified homotopy link of $Y$ in $X$, $\operatorname{denoted~}^{h o l i n k} \mathrm{~s}_{\mathrm{s}}(X, Y)$, consists of all $\omega$ in holink $(X, Y)$ such that $\omega((0,1])$ lies in a single stratum of $X$ :

$$
\operatorname{holink}_{\mathrm{s}}(X, Y)=\left\{\omega \in \operatorname{holink}(X, Y) \mid \text { for some } i, \omega(t) \subseteq X_{i} \text { for all } t \in I\right\} .
$$

The stratified homotopy link has a natural filtration with $i$-skeleton

$$
\operatorname{holink}_{\mathrm{S}}(X, Y)^{i}=\left\{\omega \mid \omega(1) \in X^{i}\right\} .
$$

The holink evaluation (at 0 ) restricts to a map $q: \operatorname{holink}_{\mathrm{s}}(X, Y) \rightarrow Y$.

Let $\mathrm{P}_{\mathrm{nsp}}(X)$ be the space of nearly stratum preserving paths in $X$; that is, those paths $\omega: I \rightarrow Y$ such that $\omega((0,1])$ lies in a single stratum of $X$. Thus,

$$
\mathrm{P}_{\mathrm{nsp}}(X)=\left\{\omega \in X^{I} \mid \omega((0,1]) \subseteq X_{i} \text { for some } i \in \mathcal{I}\right\} .
$$

Define $q: \mathrm{P}_{\mathrm{nsp}}(X) \rightarrow X$ to be evaluation at $0, q(\omega)=\omega(0)$.

There is a natural partition of $\mathrm{P}_{\mathrm{nsp}}(X)$ into disjoint subspaces

$$
\mathrm{P}_{\mathrm{nsp}}(X)_{i}=\left\{\omega \in \mathrm{P}_{\mathrm{nsp}}(X) \mid \omega(1) \in X_{i}\right\} .
$$

Throughout the rest of the paper, we will assume that $\mathrm{P}_{\mathrm{nsp}}(X)$ is endowed with this natural partition.

Define the total homotopy link of $X$ to be

$$
\operatorname{holink}(X)=\bigcup_{i \in \mathcal{I}} \operatorname{holink}_{\mathrm{s}}\left(X, X_{i}\right) \subseteq X^{I}
$$

with evaluation $q$ : holink $(X) \rightarrow X$. Naturally partition the total homotopy link by setting

$$
\operatorname{holink}(X)_{i}=\mathrm{P}_{\mathrm{nsp}}(X)_{i} \cap \operatorname{holink}(X)
$$

for $i \in \mathcal{I}$.

If $Y \subseteq X$ is a union of strata of $X$, define

$$
\mathrm{P}_{\mathrm{nsp}}(X, Y)=\operatorname{holink}_{\mathrm{s}}(X, Y) \cup \mathrm{P}_{\mathrm{nsp}}(Y)
$$

with evaluation $q: \mathrm{P}_{\mathrm{nsp}}(X, Y) \rightarrow Y$. Again the partition of $\mathrm{P}_{\mathrm{nsp}}(X)$ induces a partition of $\mathrm{P}_{\mathrm{nsp}}(X, Y)$.

Finally, define the space of stratum preserving paths in $X$ to be

$$
\mathrm{P}_{\mathrm{sp}}(X)=\left\{\omega \in X^{I} \mid \omega(I) \subseteq X_{i} \text { for some } i \in \mathcal{I}\right\} .
$$

Thus, $\mathrm{P}_{\mathrm{nsp}}(X)=\operatorname{holink}(X) \cup \mathrm{P}_{\mathrm{sp}}(X)$ and holink $(X) \cap \mathrm{P}_{\mathrm{sp}}(X)=\emptyset$. 


\section{StRATified Fibrations}

Let $X$ and $Y$ be spaces with partitions $\left\{X_{i}\right\}_{i \in \mathcal{I}}$ and $\left\{Y_{j}\right\}_{j \in \mathcal{J}}$, respectively.

Definition 5.1. A map $p: X \rightarrow Y$ is a stratified fibration provided given any space $Z$ and any commuting diagram

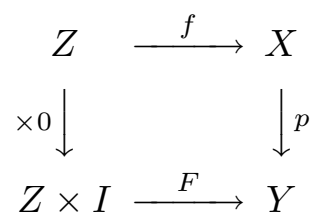

with $F$ a stratum preserving homotopy, there exists a stratified solution; i.e., a stratified homotopy $\tilde{F}: Z \times I \rightarrow X$ such that $\tilde{F}(z, 0)=f(z)$ for each $z \in Z$ and $p \tilde{F}=F$. The diagram above is a stratified homotopy lifting problem.

As an example, consider the evaluation $q: \mathrm{P}_{\mathrm{sp}}(X) \rightarrow X$. The standard proof that the evaluation $X^{I} \rightarrow X$ is a fibration shows that $q$ is a stratified fibration.

Another example occurs when a group $G$ acts discontinuously on a space $X$ such that the orbit space $X / G$ is homotopically stratified by the orbit type stratification. Then Beshears [2] has shown that under mild hypothesis, the orbit map $X \rightarrow X / G$ is a stratified fibration. Such actions include locally linear actions of finite groups on manifolds. The proof in [2] relies on some of the results in this paper.

In the usual theory of fibrations certain partial solutions can be extended [18, p. 35]. We will need a stratified version. For notation let $Z$ be a metric space with a closed subspace $A \subseteq Z$ such that the inclusion $A \rightarrow Z$ is a cofibration. Thus $(Z, A)$ is an NDR-pair and so $Z \times\{0\} \cup A \times I$ is a strong deformation retract of $Z \times I$ [18, p. 22]. Let $K: Z \times I \times I \rightarrow Z \times I$ be such a strong deformation retraction so that $K_{0}=\operatorname{id}_{Z \times I}, K_{t} \mid(Z \times\{0\} \cup A \times I)$ is the inclusion for all $t \in I$ and $K_{1}(Z \times I)=Z \times\{0\} \cup A \times I$.

Lemma 5.2 Stratified Relative Lifting. Suppose $p: X \rightarrow Y$ is a stratified fibration and there is a commuting diagram

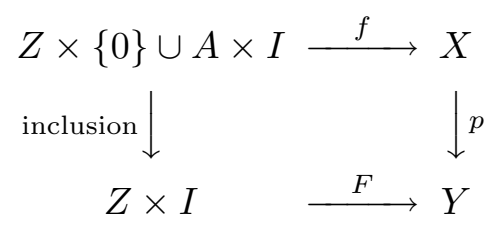

with $f$ and $F$ stratum preserving along $I$; that is, a stratified relative lifting problem. If $(Z, A)$ is a metric NDR-pair and $K: Z \times I \times I \rightarrow Z \times I$ is a strong deformation retraction as above with the additional properties:

(1) $F K: Z \times I \times I \rightarrow Y$ is stratum preserving along the second I factor,

(2) $f K_{1}: Z \times I \rightarrow X$ is stratum preserving along $I$,

then there is a stratified solution extending $f$; that is, a stratum preserving homotopy $\tilde{F}: Z \times I \rightarrow X$ such that $p \tilde{F}=F$ and $\tilde{F} \mid(Z \times\{0\} \cup A \times I)=f$.

Proof. Let $\varphi: Z \times I \rightarrow I$ be a map such that $\varphi^{-1}(0)=Z \times\{0\} \cup A \times I$ and define $H: Z \times I \times I \rightarrow Z \times I$ by

$$
H(z, s, t)= \begin{cases}K\left(z, s, 1-\frac{t}{\varphi(z, s)}\right), & \text { if } t<\varphi(z, s) \\ K(z, s, 0), & \text { if } t \geq \varphi(z, s) .\end{cases}
$$


Now

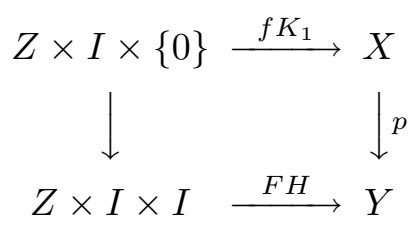

is a stratified lifting problem (that $F H$ is stratum preserving along the second $I$ factor follows from condition (1) on $F K$ above) so there is a stratified solution $G: Z \times I \times I \rightarrow X$. One checks that the homotopy $\tilde{F}: Z \times I \rightarrow X$ defined by $\tilde{F}(z, s)=G(z, s, \varphi(z, s))$ is a stratified solution of the original problem extending $f$ (that $\tilde{F}$ is stratum preserving follows from the fact that $G$ is and condition (2) on $f K_{1}$ above).

Lemma 5.3. Suppose $p: X \rightarrow Y$ is a stratified fibration and there is a stratified lifting problem

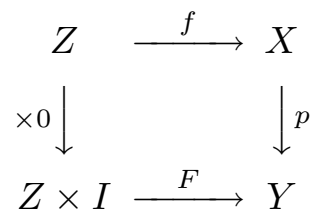

with two solutions $G, H: Z \times I \rightarrow X$. Then there exists a homotopy $J: G \simeq H$ rel $Z \times\{0\}$ such that

(1) $p J=F \times \mathrm{id}_{I}$, and

(2) $J$ is stratum preserving along $I \times I$.

Proof. The usual proof (e.g. [15, pp. 100-101]) in the unstratified case works here. Alternatively, if one is willing to assume that $Z$ is metric, then Lemma 5.2 can be applied.

Definition 5.4. A map $p: X \rightarrow Y$ is a stratified approximate fibration provided given any space $Z$ and any commuting diagram

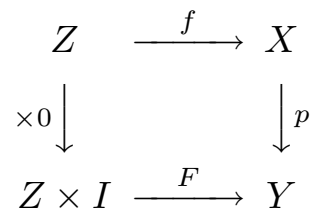

with $F$ is a stratum preserving homotopy, there exists a stratified controlled solution; i.e., a map $\tilde{F}: Z \times I \times[0,1) \rightarrow X$ which is stratum preserving along $I \times[0,1)$ such that $\tilde{F}(z, 0, t)=f(z)$ for each $(z, t) \in Z \times[0,1)$ and the function $\bar{F}: Z \times I \times I \rightarrow Y$ defined by $\bar{F} \mid Z \times I \times[0,1)=p \tilde{F}$ and $\bar{F} \mid Z \times I \times\{1\}=F \times \operatorname{id}_{\{1\}}$ is continuous and stratum preserving along $I \times I$.

Note that the partitions of $X$ and $Y$ need not be stratifications and the map $p$ need not be stratified.

\section{Remarks 5.5.}

(1) If $K \subseteq X$ is a union of a subcollection of $\left\{X_{i}\right\}_{i \in \mathcal{I}}$ and $p: X \rightarrow Y$ is a stratified fibration (or stratified approximate fibration), then so is $p \mid: K \rightarrow$ $Y$. 
(2) If $X$ and $Y$ are metric spaces, then in the definition of a stratified fibration or stratified approximate fibration $p: X \rightarrow Y$ there is no loss of generality in assuming that the spaces $Z$ in the homotopy lifting problems are metric spaces. This is because there is an universal lifting problem whose solution implies that any other problem can be solved. For the universal problem the space $Z$ is a subspace of $Y^{I} \times X$ and, hence, is metric (cf. $[9, \S 12]$ ).

The following lemma shows that we can relax the requirement in the definition of stratified approximate fibrations that stratified controlled solutions agree at all times with the given initial lift.

Lemma 5.6. Suppose

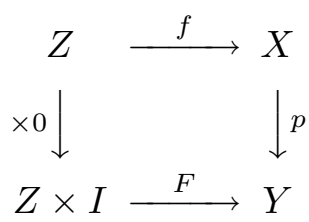

is a stratified lifting problem (i.e., the diagram commutes and $F$ is a stratum preserving homotopy) and $g: Z \times(I \times[0,1) \cup\{0\} \times I) \rightarrow X$ is a map such that

(1) $g$ is stratum preserving along $I \times[0,1) \cup\{0\} \times I$,

(2) $g(z, 0,1)=f(z)$ for each $z \in Z$, and

(3) the function $\bar{g}: Z \times I \times I \rightarrow Y$ defined by

$$
\bar{g}(z, s, t)= \begin{cases}p g(z, s, t), & \text { if } t<1 \text { or } s=0 \\ F(z, s), & \text { if } t=1\end{cases}
$$

is continuous and stratum preserving along $I \times I$.

Then there exists a stratified controlled solution $\tilde{F}: Z \times I \times[0,1) \rightarrow X$ of the given problem.

Proof. Define $\tilde{F}$ by $\tilde{F}(z, s, t)=g(x, s,(1-s)(1-t)+t)$.

\section{Statements of the main Results}

In this section we state the main results and formulate inductive statements from which the main results will follow. After more background work in $\S \S 7,8$, the proofs of the main results are completed in $\S 9$.

Theorem 6.1. If $X$ is a homotopically stratified metric space with a finite number of strata and $Y \subseteq X$ is a closed union of strata, then the evaluation map

$$
q: \mathrm{P}_{\mathrm{nsp}}(X, Y) \rightarrow Y
$$

is a stratified fibration.

As pointed out below after Theorem 6.8, Theorem 6.1 will follow from Theorem 6.7. The proof of Theorem 6.7 will be completed in $\S 9$.

Corollary 6.2. If $X$ is a homotopically stratified metric space with a finite number of strata, $Y \subseteq X$ is a closed union of strata and $X_{i} \subseteq X$ is a stratum, then each of the following evaluation maps is a stratified fibration:

(1) $q: \mathrm{P}_{\mathrm{nsp}}(X) \rightarrow X$, 
(2) $q: \operatorname{holink}_{\mathrm{s}}(X, Y) \rightarrow Y$,

(3) $q: \operatorname{holink}(X) \rightarrow X$,

(4) $q: \mathrm{P}_{\mathrm{nsp}}\left(X, X_{i}\right) \rightarrow X_{i}$,

(5) $q: \operatorname{holink}_{\mathrm{s}}\left(X, X_{i}\right) \rightarrow X_{i}$.

Proof. (1) follows from Theorem 6.1 and the equality $\mathrm{P}_{\mathrm{nsp}}(X)=\mathrm{P}_{\mathrm{nsp}}(X, X)$.

(2) follows from Theorem 6.1 and the fact that $\operatorname{holink}_{\mathrm{s}}(X, Y)$ is a union of strata of $\mathrm{P}_{\mathrm{nsp}}(X, Y)$ (see Remark 5.5(1)).

For (3), let

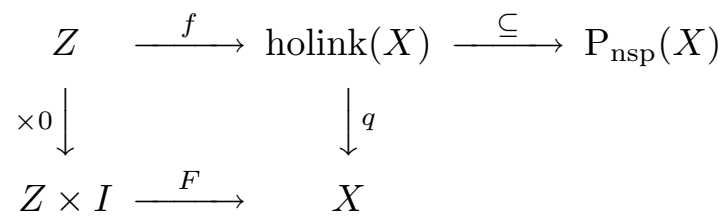

be a stratified lifting problem. From (1), there is a stratified solution in $\mathrm{P}_{\mathrm{nsp}}(X)$, $\tilde{F}: Z \times I \rightarrow \mathrm{P}_{\text {nsp }}(X)$. However, the image of $\tilde{F}$ is actually in $\operatorname{holink}(X)$. For if $(z, s) \in Z \times I$, let $X_{i}$ be the stratum of $X$ containing $F(\{z\} \times I)$ and let $X_{j}$ be the stratum containing $f(z)(0)$. Then $i \neq j$ and $f(z) \in \operatorname{holink}(X)_{j}$. Since $\tilde{F}$ is stratum preserving, $\tilde{F}(z, s) \in \operatorname{holink}(X)_{j}$ for all $s \in I$. Thus, $\tilde{F}(z, s)(t) \in X_{j}$ for all $t>0$ and $\tilde{F}(z, s)(0)=q \tilde{F}(z, s)=F(z, s) \in X_{i}$. It follows that $\tilde{F}(z, s) \in \operatorname{holink}(X)$.

Finally, note that $X_{i}$ is a closed union of strata in $\left(X \backslash X^{i}\right) \cup X_{i}$ and

$$
\begin{gathered}
\operatorname{holink}_{\mathrm{s}}\left(X, X_{i}\right)=\operatorname{holink}_{\mathrm{s}}\left(\left(X \backslash X^{i}\right) \cup X_{i}, X_{i}\right), \\
\mathrm{P}_{\mathrm{nsp}}\left(X, X_{i}\right)=\mathrm{P}_{\mathrm{nsp}}\left(\left(X \backslash X^{i}\right) \cup X_{i}, X_{i}\right) .
\end{gathered}
$$

Thus, (4) and (5) follow from Theorem 6.1 and (2), respectively.

Theorem 6.3. If $X$ is a homotopically stratified metric space with a finite number of strata and $Y \subseteq X$ is a closed union of strata, then $Y$ is stratified forward tame in $X$.

As pointed out below after Theorem 6.8, Theorem 6.3 will follow from Theorem 6.7. The proof of Theorem 6.7 will be completed in $\S 9$.

We now restate and prove the Stratum Preserving Deformation Extension Property from the introduction.

Corollary 6.4. Let $X$ be a homotopically stratified metric space with a finite number of strata and let $Y \subseteq X$ be a closed union of some of the strata of $X$. If $f: Y \times I \rightarrow Y$ is a stratum preserving deformation, then there exists a stratum preserving deformation $\tilde{f}: X \times I \rightarrow X$ extending $f$.

Proof. Let $U$ be a neighborhood of $Y$ in $X$ for which there is a nearly stratum preserving deformation $h: U \times I \rightarrow X$ of $U$ to $Y$ in $X$ rel $Y$ (by Theorem 6.3). Define a stratum preserving lifting problem

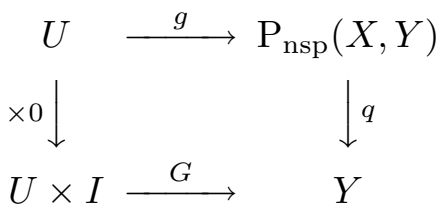


by $g(x)(t)=h(x, 1-t)$ and $G(x, t)=f(h(x, 1), t)$. By Theorem 6.1 there is a stratified solution $\tilde{G}: U \times I \rightarrow \mathrm{P}_{\text {nsp }}(X, Y)$. Let $\rho: X \rightarrow I$ be a map such that $\rho^{-1}(0)=Y$ and $\rho^{-1}(1)=X \backslash U$. Define $\tilde{f}: X \times I \rightarrow X$ by

$$
\tilde{f}(x, t)= \begin{cases}f(x, t), & \text { if } \rho(x)=0 \\ \tilde{G}(x, s)\left(\frac{\rho(x)(1-s)}{1-\rho(x)}\right), & \text { if } 0<\rho(x)<1 \text { and } \rho(x) \leq s \leq 1 \\ \tilde{G}(x, s)\left(\frac{(\rho(x)-1) s+\rho(x)}{\rho(x)}\right), & \text { if } 0<\rho(x)<1 \text { and } 0 \leq s \leq \rho(x) \\ x, & \text { if } \rho(x)=1\end{cases}
$$

where $s=t(1-\rho(x))$.

We now formulate the statements which will be proven inductively in later sections in order to deduce Theorems 6.1 and 6.3. Let $k \geq 0$ and $l \geq 1$ be integers.

Statement $S_{k, l}$. If $X$ is a homotopically stratified metric space, $Y \subseteq X$ is a closed union of strata, $X \backslash Y$ has at most $k$ strata and $Y$ has at most $l$ strata, then the evaluation map $q: \mathrm{P}_{\mathrm{nsp}}(X, Y) \rightarrow Y$ is a stratified fibration.

Statement $T_{k, l}$. If $X$ is a homotopically stratified metric space, $Y \subseteq X$ is a closed union of strata, $X \backslash Y$ has at most $k$ strata and $Y$ has at most $l$ strata, then $Y$ is stratified forward tame in $X$.

\section{Remark 6.5.}

(1) $S_{0,1}$ holds. For if $X$ has a single stratum, then $\mathrm{P}_{\mathrm{nsp}}(X, X)=X^{I}$ and evaluation at $0 X^{I} \rightarrow X$ is a fibration.

(2) $T_{0, l}$ holds for all $l \geq 1$ vacuously.

(3) $T_{1,1}$ holds by the Forward Tameness condition (Definition 3.3(i)).

The induction gets started in the following Proposition whose proof relies on the work of $[9]$ on stratified spaces with two strata.

Proposition 6.6. $S_{1,1}$ holds.

Proof. Let $X$ be a homotopically stratified metric space, let $Y \subseteq X$ be a closed union of strata and assume that $X \backslash Y$ and $Y$ are each a single stratum. In the terminology of $[9](X, Y)$ is a homotopically stratified pair. According to $[9$, Thm. 4.2] there exist a neighborhood $U$ of $Y$ in $X$ and a retraction $r: U \rightarrow Y$ such that $(X, Y)$ has the $W(r)$-lifting property. We now recall this property. Let

$$
W(r)=\left\{(x, \omega) \in U \times Y^{I} \mid r(x)=\omega(1)\right\} .
$$

The $W(r)$-lifting property asserts the existence of a map $\alpha: W(r) \rightarrow X^{I}$ such that

(1) $\alpha(x, \omega)(0)=\omega(0)$ for each $(x, \omega) \in W(r)$,

(2) $\alpha(x, \omega)(1)=x$ for each $(x, \omega) \in W(r)$,

(3) if $x \in Y$, then $\alpha(x, \omega)=\omega$,

(4) if $x \in U \backslash Y$, then $\alpha(x, \omega) \in \operatorname{holink}(X, Y)$.

Now consider a lifting problem

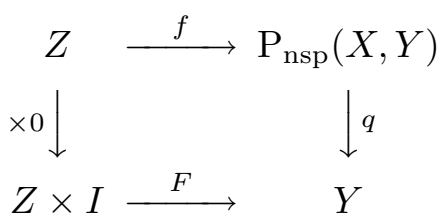


According to Remark 5.5 we may assume that $Z$ is metric. Using a partition of unity one can construct a map

$$
\epsilon: Z \rightarrow(0,1]
$$

such that for every $z \in Z$ and $0 \leq t \leq \epsilon(z)$, we have $f(z)(t) \in U$. Define a map $\omega: Z \times I \rightarrow Y^{I}$ by

$$
\omega(z, t)(s)= \begin{cases}F(z, t-2 t s), & \text { if } 0 \leq s \leq 1 / 2 \\ r(f(z)(\epsilon(z)(2 t s-t))), & \text { if } 1 / 2 \leq s \leq 1 .\end{cases}
$$

Note that $\omega(z, 0)(s)=F(z, 0)=f(z)(0)$ for all $z \in Z$ and $s \in I$. Now define

$$
\delta: Z \times I \rightarrow X^{I} \text { by } \delta(z, t)=\alpha(f(z)(\epsilon(z) t), \omega(z, t))
$$

and note that

(1) $\delta(z, 0)(s)=F(z, 0)$,

(2) $\delta(z, t)(1)=f(z)(\epsilon(z) t)$,

(3) $\delta(z, t)(0)=F(z, t)$.

(4) if $f(z) \in Y^{I}$, then $\delta(z, t) \in Y^{I}$.

Finally, define a stratified solution $\tilde{F}: Z \times I \rightarrow \mathrm{P}_{\text {nsp }}(X, Y)$ of the lifting problem by

$$
\tilde{F}(z, t)(s)= \begin{cases}\delta(z, t)(s / \epsilon(z) t), & \text { if } 0 \leq s<\epsilon(z) t \\ f(z)(s), & \text { if } \epsilon(z) t \leq s \leq 1\end{cases}
$$

Theorem 6.7. Let $k \geq 0$ and $l \geq 0$ be integers.

(1) $T_{l-1,1}, T_{k, 1}, T_{k, l-1}$ and $S_{k, l-1}$ imply $T_{k, l}$ if $l>1$.

(2) $T_{k-1,1}$ and $S_{k-1,1}$ imply $S_{k, 1}$ if $k>1$.

(3) $T_{k-1,1}$ and $S_{k-1,1}$ imply $T_{k, 1}$ if $k>1$.

(4) $T_{k+l, 1} S_{k+l, 1}$ and $S_{k, l}$ imply $S_{k, l+1}$.

Theorem 6.8. If Theorem 6.7 holds, then Statements $S_{k, l}$ and $T_{k, l}$ hold whenever $k, l \geq 0$ and $k+l \geq 1$.

Proof. The proof is by induction on $k+l$. Assume $k+l=1$ and note that $S_{0,1}$ and $T_{0,1}$ hold by Remark 6.5. Since $S_{1,0}$ and $T_{0,1}$ are empty statements, we may proceed. Assume inductively that $k+l>1$ and that $S_{a, b}$ and $T_{a, b}$ hold whenever $a, b \geq 0$ and $1 \leq a+b<k+l$.

We begin by verifying $T_{k, l}$. Consider first the case $l=1$. Then $k \geq 1$. As observed in Remark 6.5, $T_{1,1}$ holds. Thus, assume $l=1$ and $k>1$. Then $(k-1)+$ $1=k<k+l$, so we have Statements $T_{k-1,1}$ and $S_{k-1,1}$. Now $T_{k, 1}$ follows from Theorem 6.7(3).

Consider now the case $l>1$ (and $k \geq 1$ ). Then $(l-1)+1=l<k+l, k+1<k+l$ and $k+(l-1)<k+l$. Thus we have Statements $T_{l-1,1}, T_{k, 1}, T_{k, l-1}$ and $S_{k, l-1}$. Now $T_{k, l}$ follows from Theorem 6.7(1).

Hence we have verified $T_{k, l}$ and we may assume that $T_{a, b}$ holds whenever $a, b \geq 0$ and $1 \leq a+b \leq k+l$.

We now verify $S_{k, l}$. Consider first the case $l=1$. Then $k \geq 1$. Note that $S_{1,1}$ follows from Proposition 6.6. Thus, we may assume that $l=1$ and $k>1$. Then 
$(k-1)+1=k \leq k+l$ and $(k-1)+1=k<k+l$. Thus, we have $T_{k-1,1}$ and $S_{k-1,1}$. Now $S_{k}, 1$ follows from Theorem 6.7(2).

Now consider the case $l>1$. Then $(k+l-1)+1=k+l$ so $T_{k+l-1,1}$ holds. We know that $S_{k+l-1,1}$ holds from the case above. And $k+(l-1)<k+l$ so $S_{k, l-1}$ holds. Now $S_{k, l}$ follows from Theorem 6.7(4).

Note that Theorems 6.1 and 6.3 follow immediately. The missing link is the proof of Theorem 6.7 which will be completed in $\S 9$.

\section{STRATIFIED SYSTEMS OF STRATIFIED FIBRATIONS}

Quinn [13] introduced stratified systems of fibrations over stratified spaces. We now generalize this to stratified systems of stratified fibrations in which the domain as well as the range is stratified.

In this section our minimal standing hypothesis will be that $X$ denotes a space with a partition $\left\{X_{i}\right\}_{i \in \mathcal{I}}$ and $Y$ denotes a space with a filtration $\left\{Y^{j}\right\}_{j \in \mathcal{J}}$ such that $Y$ is partitioned by its strata $\left\{Y_{j}\right\}_{j \in \mathcal{J}}$.

Definition 7.1. If $p: X \rightarrow Y$ is a map and $A \subseteq Y$, then $A$ is said to be a stratified $p$-NDR subset of $Y$ if there exist a neighborhood $U$ of $A$ in $Y$ and a strong deformation retraction of $U$ to $A$ in $Y$ which is covered by a stratum preserving strong deformation retraction of $p^{-1}(U)$ to $p^{-1}(A)$ in $X$; that is, there exist homotopies $h: U \times I \rightarrow Y$ and $\tilde{h}: p^{-1}(U) \times I \rightarrow X$ such that

(1) $h(y, 0)=y$ and $\tilde{h}(x, 0)=x$ for all $y \in U, x \in p^{-1}(U)$,

(2) $h(y, t)=y$ and $\tilde{h}(x, t)=x$ for all $(y, t) \in A \times I,(x, t) \in p^{-1}(A) \times I$,

(3) $h(y, 1) \in A$ and $\tilde{h}(x, 1) \in p^{-1}(A)$ for all $y \in U, x \in p^{-1}(U)$,

(4) $p \tilde{h}(x, t)=h(p(x), t)$ for all $x \in p^{-1}(U), t \in I$,

(5) $\tilde{h}$ is a stratum preserving homotopy.

Special attention should be paid to the condition in the definition above that $\tilde{h}$ is required to be stratum preserving, not just nearly stratum preserving. In particular, consider the identity map $\operatorname{id}_{X}: X \rightarrow X$. If both the domain and range are given the same filtration, then the skeleta of $X$ are not, in general, $\mathrm{id}_{X}$-NDR subsets of $X$. However, if the domain is unstratified (i.e., consists of a single stratum) and the skeleta in the range are neighborhood strong deformation retracts, then the skeleta are $i d_{X}$-NDR subsets of $X$.

Lemma 7.2. If $Y$ is a metric space, $A \subseteq Y$ is a closed union of strata and stratified forward tame in $Y$, and $q: \mathrm{P}_{\mathrm{nsp}}(Y) \rightarrow Y$ is evaluation, then $A$ is a stratified $q$-NDR subset of $Y$.

Proof. Let $U$ be a neighborhood of $A$ in $Y$ for which there exists a nearly stratum preserving strong deformation retraction $h: U \times I \rightarrow Y$ of $U$ to $A$ in $Y$ as in Definition 3.4. Since $\mathrm{P}_{\mathrm{nsp}}(Y)$ is paracompact, there exists a map $\alpha: q^{-1}(U) \rightarrow(0,1]$ such that $\omega([0, \alpha(\omega)]) \subseteq U$ for each $\omega \in q^{-1}(U)$. Define $\tilde{h}: q^{-1}(U) \times I \rightarrow \mathrm{P}_{\mathrm{nsp}}(Y)$ by

$$
\tilde{h}(\omega, s)(t)= \begin{cases}h(\omega(2 t \alpha(\omega)), s-2 t), & \text { if } 0 \leq t \leq \frac{s}{2} \\ \omega\left(\frac{2(1-s \alpha(\omega)) t+2 s \alpha(\omega)-s}{2-s}\right), & \text { if } \frac{s}{2} \leq t \leq 1\end{cases}
$$


Corollary 7.3. If $Y$ is a metric space, $B \subseteq A \subseteq Y$ are closed unions of strata, $B$ is stratified forward tame in $Y$, and $q: \mathrm{P}_{\mathrm{nsp}}(Y, A) \rightarrow A$ is evaluation, then $B$ is a stratified $q$-NDR subset of $A$.

Proof. Let $p: \mathrm{P}_{\mathrm{nsp}}(Y) \rightarrow Y$ be evaluation. Lemma 7.2 implies that $B$ is a stratified $p$-NDR subset of $Y$. Let $U$ be a neighborhood of $B$ in $Y$ for which there exists homotopies $h: U \times I \rightarrow Y$ and $\tilde{h}: p^{-1}(U) \times I \rightarrow \mathrm{P}_{\mathrm{nsp}}(Y)$ as in 7.1. By the proof of 7.2 we may assume that $h$ is nearly stratum preserving. Note that $\mathrm{P}_{\text {nsp }}(Y, A) \subseteq$ $\mathrm{P}_{\mathrm{nsp}}(Y)$ and, in fact, $\mathrm{P}_{\mathrm{nsp}}(Y, A)_{i} \subseteq \mathrm{P}_{\mathrm{nsp}}(Y)_{i}$ for all $i$. Also $q^{-1}(B)=p^{-1}(B)$. It then follows from the explicit construction in 7.2 that $\tilde{h} \mid: q^{-1}(U) \times I \rightarrow \mathrm{P}_{\mathrm{nsp}}(Y, A)$ is a stratum preserving strong deformation retraction covering $h$.

Definition 7.4. A map $p: X \rightarrow Y$ is a stratified system of stratified fibrations if for each $j \in \mathcal{J}$,

(1) $p \mid: p^{-1}\left(Y_{j}\right) \rightarrow Y_{j}$ is a stratified fibration for each stratum $Y_{j}$ of $Y$, and

(2) each skeleton $Y^{j}$ of $Y$ is a stratified $p$-NDR subset of $Y$.

If in the definition above, $X$ is unstratified (i.e., the partition of $X$ consists of a single stratum) and $p \mid: p^{-1}\left(Y_{j}\right) \rightarrow Y_{j}$ is a fibration for each stratum $Y_{j}$ of $Y$, then $p$ is said to be a stratified system of fibrations. This notion was defined by Quinn [13] and is useful in the theory of group actions. Talbert [16] observes that any map with a homotopy colimit structure is a stratified system of fibrations.

Our interest in stratified system of fibrations is that they are usually stratified approximate fibrations. See Corollary 7.6. This is a generalization of the analogous fact for stratified systems of fibrations due to Quinn [14, 3.3]

Lemma 7.5. Let $X$ be a metric space with a partition, $Y$ a metric space with a stratification satisfying the Frontier Condition, and let $Y^{0}$ be a minimal skeleton of $Y$ (so that $\left.Y^{0}=Y_{0}\right)$. If $p: X \rightarrow Y$ is a map such that $p \mid: p^{-1}\left(Y \backslash Y^{0}\right) \rightarrow Y \backslash Y^{0}$ is a stratified approximate fibration, $p \mid: p^{-1}\left(Y^{0}\right) \rightarrow Y^{0}$ is a stratified fibration, and $Y^{0}$ is a stratified $p$-NDR subset of $Y$, then $p: X \rightarrow Y$ is a stratified approximate fibration.

Proof. Let $U$ be a neighborhood of $Y^{0}$ in $Y$ for which there exists a strong deformation retraction $h: U \times I \rightarrow Y$ of $U$ to $Y^{0}$ in $Y$ which is covered by a stratum preserving strong deformation retraction $\tilde{h}: p^{-1}(U) \times I \rightarrow X$ of $p^{-1}(U)$ to $p^{-1}\left(Y^{0}\right)$ in $X$ as in Definition 7.1. Suppose there is given a stratified lifting problem

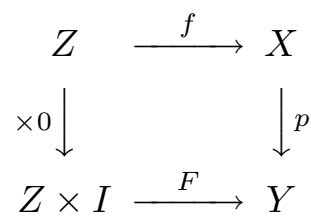

for which we need to find a stratified controlled solution. According to Remark 5.5(2) we may assume that $Z$ is a metric space. Let $Z_{0}=F_{0}^{-1}\left(Y^{0}\right)$. Since $F$ is stratum preserving, $Z_{0} \times I=F^{-1}\left(Y^{0}\right)$. Let $Z_{1}=F_{0}^{-1}\left(Y \backslash Y^{0}\right)$. Since $F$ is stratum preserving, $Z_{1} \times I=F^{-1}\left(Y \backslash Y^{0}\right)$. Thus, there is a stratified lifting problem

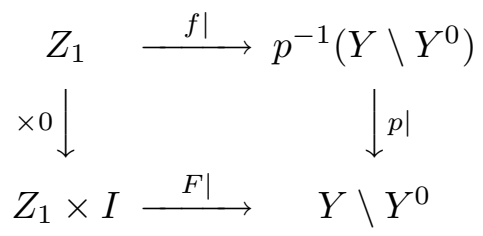


and, since $p \mid: p^{-1}\left(Y \backslash Y^{0}\right) \rightarrow Y \backslash Y^{0}$ is a stratified approximate fibration, there is a stratified controlled solution

$$
g: Z_{1} \times I \times[0,1) \rightarrow p^{-1}\left(Y \backslash Y^{0}\right) .
$$

Thus $g(z, 0, t)=f(z)$ for all $(z, t) \in Z_{1} \times[0,1), g$ is stratum preserving along $I \times[0,1)$, and the function $\bar{g}: Z_{1} \times I \times I \rightarrow Y \backslash Y^{0}$ defined by

$$
\bar{g}(z, s, t)= \begin{cases}p g(z, s, t), & \text { if }(z, s, t) \in Z_{1} \times I \times[0,1) \\ F(z, s), & \text { if }(z, s) \in Z_{1} \times I \text { and } t=1\end{cases}
$$

is continuous and stratum preserving along $I \times I$. Choose a neighborhood $Z^{\prime}$ of $Z_{0}$ in $Z$ such that $F\left(Z^{\prime} \times I\right) \subseteq U$ and let $Z_{1}^{\prime}=Z^{\prime} \cap Z_{1}=Z^{\prime} \backslash Z_{0}$. Use the fact that $Z$ is paracompact to define a map $\alpha: Z_{1}^{\prime} \rightarrow[0,1)$ such that $\bar{g}(z, s, t) \in U$ if $\alpha(z, s) \leq t \leq 1$ and let $\tilde{\alpha}: Z \rightarrow I$ be any continuous extension of $\alpha$. Let $\rho: Z \rightarrow I$ be a map such that $\rho^{-1}(0)=Z_{0}$ and $\rho\left(Z \backslash Z^{\prime}\right)=1$. Define $\beta: Z^{\prime} \times I \rightarrow Y$ by

$$
\beta(z, s)= \begin{cases}h(\bar{g}(z, s, \rho(z) \cdot \alpha(z, s)+1-\rho(z)), 1), & \text { if }(z, s) \in Z_{1}^{\prime} \times I \\ F(z, s), & \text { if }(z, s) \in Z_{0} \times I,\end{cases}
$$

define $\tilde{\beta}: Z_{1}^{\prime} \times I \rightarrow X$ by

$$
\tilde{\beta}(z, s)=\tilde{h}(g(z, s, \rho(z) \cdot \alpha(z, s)+1-\rho(z)), 1)
$$

and note that $p \circ \tilde{\beta}=\beta \mid Z_{1}^{\prime} \times I$. Define $A \subseteq Z^{\prime} \times[0,1)$ by $A=\{(z, t) \mid \rho(z) \geq$ $\left.1-t, z \in Z^{\prime}\right\}$ and let

$$
B=\left\{(z, s, t) \mid \rho(z) \geq 1-t, s \in I, z \in Z^{\prime}\right\} \subseteq Z^{\prime} \times I \times[0,1),
$$

so that with a slight abuse of notation, $B$ can be identified with $A \times I$. See Figure 7.5.1. 
We will now show that $Z^{\prime} \times\{0\} \times[0,1) \cup B$ is a strong deformation retract of $Z^{\prime} \times I \times[0,1)$. First we need an auxiliary map. Given $0 \leq \tau \leq 1$ define $R_{\tau}: I \times I \times I \rightarrow I$ by

$$
R_{\tau}(s, t, u)= \begin{cases}(s-u s, t+u s), & \text { if } t \leq \tau-s \\ (s-u t-u \tau, t-u t-u \tau), & \text { if } \tau-s \leq t \leq \tau \\ (s, t), & \text { if } \tau \leq t\end{cases}
$$

Thus $R_{\tau}$ is a strong deformation retraction of $I \times I$ onto $\{(s, t) \in I \times I \mid s=$ 0 or $\tau \leq t\}$. Now define $K: Z^{\prime} \times I \times[0,1) \times I \rightarrow Z^{\prime} \times I \times[0,1)$ by $K(z, s, t, u)=$ $\left(z, R_{1-\rho(z)}(s, t, u)\right)$. Thus, $K$ is a strong deformation retraction of $Z^{\prime} \times I \times[0,1)$ onto $Z^{\prime} \times\{0\} \times[0,1) \cup B$. Define $\gamma: Z^{\prime} \times\{0\} \times[0,1) \cup B \rightarrow p^{-1}\left(Y^{0}\right)$ by

$$
\gamma(z, s, t)= \begin{cases}\tilde{h}(f(z), 1), & \text { if }(z, s, t) \in Z^{\prime} \times\{0\} \times[0,1) \\ \tilde{\beta}(z, s), & \text { if }(z, s, t) \in B .\end{cases}
$$

Define $\Gamma: Z^{\prime} \times I \times[0,1) \rightarrow Y^{0}$ by $\Gamma(z, s, t)=\beta(z, s)$ and note that

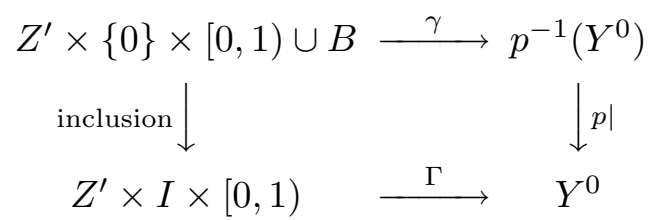

commutes. In fact, $\gamma$ and $\Gamma$ are stratum preserving along $I$ and the strong deformation $K$ is such that

(1) $\Gamma K: Z^{\prime} \times I \times[0,1) \times I \rightarrow Y^{0}$ is stratum preserving along the final $I$ factor (because $Y^{0}$ has only a single stratum!), and

(2) $\gamma K_{1}: Z \times I \times[0,1) \rightarrow p^{-1}\left(Y^{0}\right)$ is stratum preserving along $I$ (this requires a check of the definitions).

Thus Lemma 5.2 implies that there is a stratified solution

$$
\tilde{\Gamma}: Z^{\prime} \times I \times[0,1) \rightarrow p^{-1}\left(Y^{0}\right)
$$

extending $\gamma$; that is,

(1) $\tilde{\Gamma}$ is stratum preserving along $I$,

(2) $\tilde{\Gamma}\left|Z^{\prime} \times\{0\} \times[0,1)=\gamma\right|$,

(3) $\tilde{\Gamma}|B=\gamma|$,

(4) $p \tilde{\Gamma}=\Gamma$.

See Figure 7.5.2.

Define $\Lambda: Z \times(I \times[0,1) \cup\{0\} \times I) \rightarrow X$ by

$$
\Lambda(z, s, t)=\left\{\begin{array}{lc}
\tilde{\Gamma}(z, s, t), & \text { if } t \leq 1-\rho(z), z \in Z^{\prime}, t<1 \\
\tilde{h}\left(g(z, s,(1-t) \alpha(z, s)+t), \frac{1-t}{\rho(z)}\right), & \text { if } t \geq 1-\rho(z), z \in Z^{\prime}, t<1 \\
& (\text { i.e. }(z, s, t) \in B) \\
g(z, s,(1-t) \tilde{\alpha}(z, s)+t), & \text { if } z \in Z \backslash Z^{\prime}, t=1 \\
f(z), & \text { if } s=0, t=1 .
\end{array}\right.
$$


Define $\bar{\Lambda}: Z \times I \times I \rightarrow Y$ by

$$
\bar{\Lambda}(z, s, t)= \begin{cases}p \Lambda(z, s, t), & \text { if } t<1 \text { or } s=0 \\ F(z, s), & \text { if } t=1\end{cases}
$$

One checks that $\Lambda$ is stratum preserving along $I \times[0,1) \cup\{0\} \times I$ and $\bar{\Lambda}$ is continuous and stratum preserving along $I \times I$. Apply Lemma 5.6 to turn $\Lambda$ into a controlled stratified solution of the original problem.

Corollary 7.6. Let $X$ be a metric space with a partition, $Y$ a metric space with a stratification satisfying the Frontier Condition such that $Y$ has only finitely many strata. If $p: X \rightarrow Y$ is a stratified system of stratified fibrations, then $p$ is a stratified approximate fibration.

Proof. This follows from Lemma 7.5 by induction on the number of strata of $Y$.

Remark 7.7. If $p: X \rightarrow Y$ is an algebraic map between algebraic varieties, then $X$ and $Y$ have Whitney stratifications with the property that $p$ takes each stratum of $X$ submersively into some stratum of $Y$. I conjecture that such maps are stratified approximate fibrations, I don't know if they are stratified fibrations, and suspect they need not be stratified systems of fibrations.

\section{Preliminary constructions}

This section contains a collection of technical results which will be needed in the proofs of the main results in $\S 9$.

Lemma 8.1 (The Limbo). Suppose $X$ is a space, $Z$ is a metric space, $A \subseteq Z$ is 
a closed subspace and there is a commuting diagram

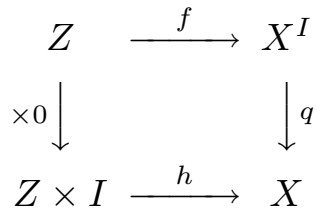

with $q(\omega)=\omega(0)$ for all $\omega \in X^{I}$ and $f(a)(t)=f(a)(0)$ for all $(a, t) \in A \times I$. Suppose also that there exists a partial lift $\tilde{h}:(Z \backslash A) \times[0,1) \rightarrow X^{I}$; that is, $q \tilde{h}=h \mid(Z \backslash A) \times[0,1)$ and $\tilde{h}(z, 0)=f(z)$ for all $z \in Z \backslash A$. Then there exists a map $u:(Z \backslash A) \times I \rightarrow I$ such that $u(z, 0)=1$ for all $z \in Z \backslash A, u^{-1}(0)=(Z \backslash A) \times\{1\}$, and so that the function $\hat{h}: Z \times I \rightarrow X^{I}$ given by

$$
\hat{h}(z, s)(t)= \begin{cases}\tilde{h}(z, s(1-t))(s u(z, 1-t)+(1-s) t), & \text { if } t>0 \text { and } z \in Z \backslash A \\ h(z, s(1-t)), & \text { if } t=0 \text { or } z \in A\end{cases}
$$

is continuous. Moreover, $\hat{h}_{0}=f$ and $q \hat{h}=h$.

Proof. For each point $(z, t) \in(Z \backslash A) \times[0,1)$ choose a number $N(z, t)$ such that

(1) $0<N(z, t) \leq 1-t$,

(2) $\operatorname{diam}\{\tilde{h}(z, t)(s) \mid 0 \leq s \leq N(z, t)\}<2 \operatorname{diam}\{f(z)(s) \mid 0 \leq s \leq t\}$,

(3) $N(z, 0)=1$ for all $z \in Z \backslash A$.

For each $(z, t) \in(Z \backslash A) \times[0,1)$ choose a neighborhood $U_{(z, t)}$ of $(z, t) \in(Z \backslash A) \times[0,1)$ such that

(1) $\operatorname{diam}\left\{\tilde{h}\left(z^{\prime}, t^{\prime}\right)(s) \mid 0 \leq s \leq N(z, t)\right\}<2 \operatorname{diam}\left\{f\left(z^{\prime}\right)(s) \mid 0 \leq s \leq t^{\prime}\right\}$ for all $\left(z^{\prime}, t^{\prime}\right) \in U_{(z, t)}$

(2) $U_{(z, t)} \cap Z \times\{0\} \neq \emptyset$ if and only if $t=0$.

Let $\left\{U_{\alpha}\right\}$ be a locally finite refinement of $\left\{U_{(z, t)}\right\}$ and let $\left\{\phi_{\alpha}\right\}$ be a partition of unity subordinate to $\left\{U_{\alpha}\right\}$. For each $\alpha$ choose $(z, t)$ such that $U_{\alpha} \subseteq U_{(z, t)}$ and set $\delta_{\alpha}=N(z, t)$. Define $u:(Z \backslash A) \times I \rightarrow I$ by $u \mid(Z \backslash A) \times[0,1)=\sum \delta_{\alpha} \phi_{\alpha}$ and $u(z, 1)=0$ for all $z \in Z \backslash A$. Note that if $z \in Z \backslash A$ and $t<1$, then $u(z, t)>0$. One checks that the function $\hat{h}$ defined above is continuous. Finally, it is easy to verify that $\hat{h}_{0}=f$ and $q \hat{h}=h$.

Here is some explanation for the preceding lemma. Consider the map $\Delta_{u}: Z \rightarrow$ $X^{I}$ defined by

$$
\Delta_{u}(z)(t)=\hat{h}(z, 1)(t)= \begin{cases}\tilde{h}(z, 1-t)(u(z, 1-t)), & \text { if } t>0 \text { and } z \in Z \backslash A \\ h(z, 1-t), & \text { if } t=0 \text { or } z \in A .\end{cases}
$$

Then $\hat{h}$ is a homotopy from $f$ to $\Delta_{u}$. One should think of $\Delta_{u}$ as the $u$-damped diagonal map. The point of the lemma is that the undamped diagonal function $\Delta: Z \rightarrow X^{I}$ defined by

$$
\Delta(z)(t)= \begin{cases}\tilde{h}(z, 1-t)(t), & \text { if } t>0 \text { and } z \in Z \backslash A \\ h(z, 1-t), & \text { if } t=0 \text { or } z \in A\end{cases}
$$

need not be continuous. For if $\left\{z_{n}\right\}$ is a sequence in $Z \backslash A$ converging to $a \in A$, there is no reason for $\left\{\tilde{h}\left(z_{n}, 1-t\right)(t)\right\}$ to converge to $h(a, 1-t)$ if $0<t<1$. But $u$ 
Figure 8.1.1. The Limbo

is chosen so that $\left\{\tilde{h}\left(z_{n}, 1-t\right)(u(z, 1-t))\right\}$ converges to $h(a, 1-t)$. The name 'The Limbo' refers to the way one must duck below the diagonal as in the Limbo dance. See Figure 8.1.1.

Addendum 8.2. In the situation of Lemma 8.1, suppose further that $X$ is a metric space with a stratification satisfying the Frontier Condition, $f(Z) \subseteq \mathrm{P}_{\mathrm{nsp}}(X), h$ is a stratum preserving homotopy so that

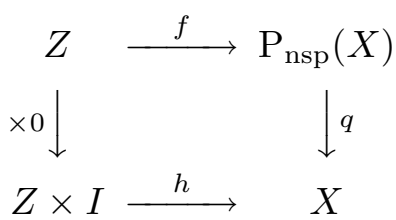

is a stratified lifting problem, and that $\tilde{h}$ has image in $\mathrm{P}_{\mathrm{nsp}}(X)$ and is stratum preserving along $[0,1)$. Then the map $\hat{h}$ defined in Lemma 8.1 has image in $\mathrm{P}_{\mathrm{nsp}}(X)$ and is a stratum preserving homotopy.

Proof. If $z \in Z$, then $\hat{h}(z, 0)=f(z) \in \mathrm{P}_{\mathrm{nsp}}(X)$. Choose $i$ such that $\hat{h}(z, 0) \in$ $\mathrm{P}_{\mathrm{nsp}}(X)_{i}$. Thus, $\hat{h}(z, 0)(t) \in X_{i}$ for each $t \in(0,1]$. We must show that $\hat{h}(z, s) \in$ $\mathrm{P}_{\text {nsp }}(X)_{i}$ for each $s \in I$; that is, we must show that $\hat{h}(z, s)(t) \in X_{i}$ for each $s \in I$ and $t \in(0,1]$. First assume that $z \in A$. Then $\hat{h}(z, s)(t)=h(z, s(1-t))$. Since $h(z, 0)=\hat{h}(z, 0)(1) \in X_{i}$ and $h$ is stratum preserving, it follows that $\hat{h}(z, s)(t)=$ $h(z, s(1-t)) \in X_{i}$ for each $s \in I$ and $t \in I$. Now assume that $z \in Z \backslash A, s \in I$ and $t \in$ $(0,1]$. Then $\hat{h}(z, s)(t)=\tilde{h}(z, s(1-t))(v)$ where $v=s u(z, 1-t)+(1-s) t$. Since $t>0$ and $u(z, 1-t)>0$, it follows that $v>0$. Since $\tilde{h}(z, 0)-f(z)=\hat{h}(z, 0) \in \mathrm{P}_{\mathrm{nsp}}(X)_{i}$, and $\tilde{h}$ is stratum preserving along $[0,1)$, it follows that $\tilde{h}(z, s(1-t)) \in \mathrm{P}_{\mathrm{nsp}}(X)_{i}$. Thus, $\tilde{h}(z, s(1-t))(v) \in X_{i}$ as required. 
Lemma 8.3. Let $X$ be a metric space with a stratification satisfying the Frontier Condition and let $K$ and $L$ be closed unions of strata of $X$ with $M=K \cap L$. Let $L^{\prime}=\left\{\omega \in X^{I} \mid \omega(t)=\omega(0) \in L\right.$ for each $\left.t \in I\right\}$. If the evaluation at 0

$$
q: \mathrm{P}_{\mathrm{nsp}}(X \backslash L, K \backslash M) \rightarrow K \backslash M
$$

is a stratified fibration, then so is the evaluation at 0

$$
q^{\prime}: \mathrm{P}_{\mathrm{nsp}}(X \backslash L, K \backslash M) \cup L^{\prime} \rightarrow K \cup L .
$$

Proof. Suppose there is given a stratified lifting problem

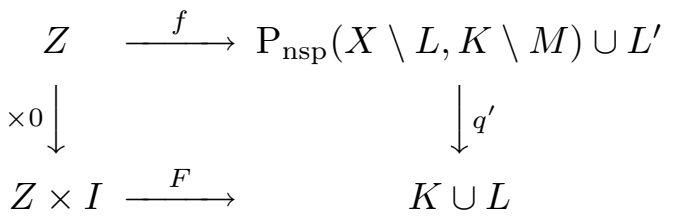

for which we need to find a stratified stratified solution. Note that $A=f^{-1}\left(L^{\prime}\right)$ is a closed subset of $Z$ and, since $F$ is stratum preserving, $F^{-1}(K \backslash M)=(Z \backslash A) \times I$. Thus, there is a stratified lifting problem

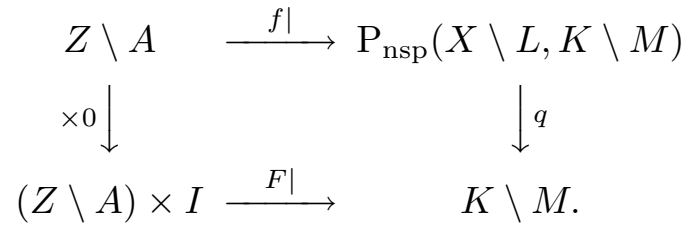

Since $q$ is a stratified fibration, there is a controlled solution $\tilde{F}:(Z \backslash A) \times I \rightarrow$ $\mathrm{P}_{\mathrm{nsp}}(X \backslash L, K \backslash M)$. According to the Limbo Lemma 8.1 there exists a map $u$ : $(Z \backslash A) \times I \rightarrow I$ such that $u(z, 0)=1, u(z, 1)=0$ for each $z \in Z \backslash A$ and so that the function $\hat{F}: Z \times I \rightarrow X^{I}$ given by

$$
\hat{F}(z, s)(t)= \begin{cases}\tilde{F}(z, s(1-t))(s u(z, 1-t)+(1-s) t), & \text { if } t>0 \text { and } z \in Z \backslash A \\ F(z, s(1-t)), & \text { if } t=0 \text { or } z \in A\end{cases}
$$

is continuous. Lemma 8.1 implies that $\hat{F}$ is a solution of the problem and Addendum 8.2 implies that $\hat{F}$ is a stratum preserving homotopy.

Lemma 8.4. Let $X$ be a metric space with a stratification satisfying the Frontier Condition and let $K$ and $L$ be closed unions of strata of $X$ with $M=K \cap L$. If $K \backslash M$ is stratified forward tame in $X \backslash L$, then there exists an open neighborhood $U$ of $K \backslash M$ in $X \backslash L$ such that $K$ is stratified forward tame in $U \cup M$.

Proof. Let $W$ be a neighborhood of $K \backslash M$ in $X \backslash L$ for which there exists a nearly stratum preserving homotopy $h: W \times I \rightarrow X \backslash L$ showing that $K \backslash M$ is stratified forward tame in $X \backslash L$ as in Definition 3.4. For each $n=1,2,3, \ldots$ let $W_{n}$ be an open neighborhood of $K \backslash M$ in $X \backslash L$ such that $\operatorname{diam} h(\{x\} \times I)<1 / n$ for each $x \in W_{n}$. Let

$$
U=\bigcup_{n=1}^{\infty}[B(1 / n, M) \backslash \operatorname{cl} B(1 /(n+1), M)] \cap W_{n} \cup[W \backslash \operatorname{cl} B(1, M)]
$$

where $B(k, M)$ denotes the set of points which are a distance less than $k$ from some point of $M$. Let $U^{\prime}$ be an open neighborhood of $K \backslash M$ in $X \backslash L$ such that $h\left(U^{\prime} \times I\right) \subseteq U$. Since $h \mid U^{\prime} \times I$ extends continuously via the identity to $M \times I$, the result follows. 
Proposition 8.5 (Blending). Let $X$ be a metric space with a stratification satisfying the Frontier Condition. Let $K$ and $L$ be closed unions of strata of $X$ such that

(1) $M=K \cap L$ is stratified forward tame in $K$,

(2) $K \backslash M$ is stratified forward tame in $X \backslash L$,

(3) $L$ is stratified forward tame in $(X \backslash K) \cup M$,

(4) $q: \mathrm{P}_{\mathrm{nsp}}(X \backslash L, K \backslash M) \rightarrow K \backslash M$ is a stratified fibration.

Then $K \cup L$ is stratified forward tame in $X$.

Proof. The stratified forward tameness conditions in items (1)-(3) above imply that there exist open neighborhoods $U_{M}$ of $M$ in $K, U_{K}$ of $K \backslash M$ in $X \backslash L$, and $U_{L}$ of $L$ in $(X \backslash K) \cup M$ together with nearly stratum preserving homotopies

(1) $h^{M}: U_{M} \times I \rightarrow K$,

(2) $h^{K}: U_{K} \times I \rightarrow X \backslash L$, and

(3) $h^{L}: U_{L} \times I \rightarrow(X \backslash K) \cup M$

as in Definition 3.4. See Figure 8.5.1

FiguRE 8.5.1

By Lemma 8.4 we can assume that $h^{K}$ is defined on $U_{K} \cup M$ and $h^{K}:\left(U_{K} \cup\right.$ $M) \times I \rightarrow X$ is such that $h^{K}(x, t)=x$ for each $(x, t) \in M \times I$. Now extend $h^{K}$ (but continue to denote it the same) to $h^{K}:\left(U_{K} \cup L\right) \times I \rightarrow X$ so that $h^{K}(x, t)=x$ for each $(x, t) \in L \times I$.

Let $\rho_{M}: K \rightarrow I$ be a map such that $\rho_{M}^{-1}(0)$ is a closed neighborhood of $M$ in $K$ and $\rho_{M}^{-1}(1)=K \backslash U_{M}$. Let $\tilde{h}^{M}:(K \cup L) \times I \rightarrow K$ be defined by

$$
\tilde{h}^{M}(x, t)= \begin{cases}h^{M}\left(x, t\left(1-\rho_{M}(x)\right)\right), & \text { if } x \in U_{M} \\ x, & \text { if } x \in\left(K \backslash U_{M}\right) \cup L .\end{cases}
$$

Note that $\tilde{h}^{M}$ deformation retracts a neighborhood of $M$ in $K$ to $M$ rel $M$ and that $\tilde{h}^{M} \mid(K \cup L) \times[0,1)$ is stratum preserving along [0,1). If $L^{\prime}=\left\{\omega \in X^{I} \mid \omega(t)=\right.$ 
$\omega(0) \in L$ for each $t \in I\}$, then it follows from Lemma 8.3 that the evaluation map $q^{\prime}: \mathrm{P}_{\text {nsp }}(X \backslash L, K \backslash M) \cup L^{\prime} \rightarrow K \cup L$ is a stratified fibration. Define $f: U_{K} \cup L \rightarrow$ $\mathrm{P}_{\mathrm{nsp}}(X \backslash L, K \backslash M) \cup L^{\prime}$ by

$$
f(x)(t)=h^{K}(x, 1-t) \quad \text { for }(x, t) \in\left(U_{K} \cup L\right) \times I .
$$

Define $F:\left(U_{K} \cup L\right) \times I \rightarrow K$ by

$$
F(x, t)=\tilde{h}^{M}\left(h^{K}(x, 1), t\right) \quad \text { for }(x, t) \in\left(U_{K} \cup L\right) \times I .
$$

Consider the commuting diagram

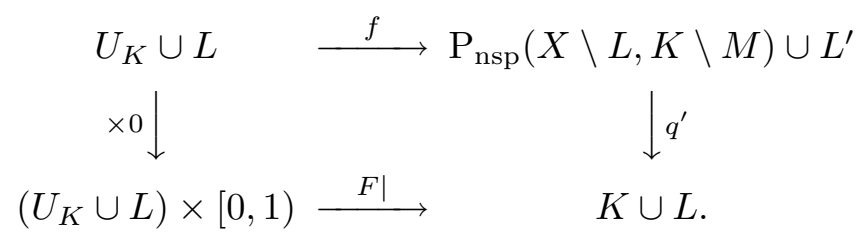

Note that $F \mid\left(U_{K} \cup L\right) \times[0,1)$ is stratum preserving along [0,1). Since $q^{\prime}$ is a stratified fibration there exists a stratified solution $\tilde{F}:\left(U_{K} \cup L\right) \times[0,1) \rightarrow \mathrm{P}_{\mathrm{nsp}}(X \backslash$ $L, K \backslash M) \cup L^{\prime}$; that is, $\tilde{F}$ is stratum preserving along $[0,1), q^{\prime} \tilde{F}=F$ and $\tilde{F}_{0}=f$. Now the Limbo Lemma 8.1 can be applied with $Z=U_{K} \cup L, A=K \cup L$, and $\tilde{F}$ the partial lift of $F$ with given initial lift $f$. It follows that there exists a map $u:\left(U_{K} \backslash K\right) \times I \rightarrow I$ such that $u(x, 0)=1, u(x, 1)=0$ for all $x \in U_{K} \backslash K$ and so that the function $\hat{F}:\left(U_{K} \cup L\right) \times I \rightarrow X$ given by

$$
\hat{F}(x, s)(t)= \begin{cases}\tilde{F}(x, s(1-t))(s u(x, 1-t)+(1-s) t), & \text { if } t>0 \text { and } x \in U_{K} \backslash K \\ F(x, s(1-t)), & \text { if } t=0 \text { or } x \in K \cup L\end{cases}
$$

is continuous. Note that $\hat{F}(x, 0)(t)=f(x)(t)=h^{K}(x, 1-t)$ for all $(x, t) \in\left(U_{K} \cup\right.$ $L) \times I$. Let $V_{K}$ be an open neighborhood of $K \backslash M$ in $X \backslash L$ such that the closure of $V_{K}$ in $X \backslash L$ is contained in $U_{K}$. Let $\rho_{K}: U_{K} \rightarrow I$ be a map such that $\rho^{-1}(0)=K \backslash M$ and $\rho_{K}^{-1}(1)=U_{K} \backslash V_{K}$. Define $F^{*}: U_{K} \cup L \rightarrow X^{I}$ by

$$
F^{*}(x)(t)= \begin{cases}\hat{F}\left(x, \rho_{K}(x)\right)(1-t), & \text { if } x \in U_{K} \\ x, & \text { if } x \in L\end{cases}
$$

In particular, $F^{*}(x)(t)=x$ for all $x \in K \cup L, F^{*}(x)(0)=x$ and $F^{*}(x)(1) \in K$ for all $x \in U_{K}$, and if $\rho_{K}(x)=1$, then $F^{*}(x)$ is a path with $F^{*}(x)(0)=x$ and $F^{*}(x)(1) \in$ $M$. Let $\rho_{L}: X \backslash(K \backslash M) \rightarrow I$ be a map such that $\rho_{L}^{-1}(0)$ is a closed neighborhood of $L$ in $X \backslash(K \backslash M)$ and $\rho_{L}^{-1}(1)=X \backslash\left(U_{L} \cup K\right)$. Let $\tilde{h}^{L}: X \backslash(K \backslash M) \times I \rightarrow X \backslash(K \backslash M)$ be defined by

$$
\tilde{h}^{L}(x, t)= \begin{cases}h^{L}\left(x, t\left(1-\rho_{L}(x)\right),\right. & \text { if } x \in U_{L} \\ x, & \text { if } x \in X \backslash U_{L} .\end{cases}
$$

Note that $\tilde{h}^{L}$ deformation retracts a neighborhood of $L$ in $X \backslash(K \backslash M)$ to $L$ rel $L$ and $\tilde{h}^{L} \mid L \times[0,1)$ is a stratum preserving along $[0,1)$. Let $\rho: X \backslash L \rightarrow I$ be a map 
Figure 8.5.2

such that $\rho^{-1}(0)$ is the closure of $V_{K}$ in $X \backslash L$ and $\rho^{-1}(1)=X \backslash\left(U_{K} \cup L\right)$. See Figure 8.5.2.

Define $H: X \times I \rightarrow X$ by

$$
H(x, t)= \begin{cases}F^{*}(x)(t), & \text { if } 0 \leq t \leq 1-\rho(x) \text { and } \rho(x) \neq 1, \\ \tilde{h}^{L}\left(F^{*}(x)(1-\rho(x)), \frac{t+\rho(x)-1}{\rho(x)}\right), & \text { or } x \in L \\ & \text { if } 1-\rho(x) \leq t \leq 1, \rho(x) \neq 0, \\ \tilde{h}^{L}(x, t), & \text { if } \rho(x)=1 \\ x, & \text { if } x \in K \cup L .\end{cases}
$$

See Figure 8.5.3.

Then $H$ deformation retracts a neighborhood of $K \cup L$ to $K \cup L$ in a way which shows that $K \cup L$ is stratified forward tame in $X$.

The following result is a generalization of a phenomenon observed by Quinn [14, 2.7], namely that approximate lifts can sometimes be turned into exact lifts.

Proposition 8.6. Let $X$ be a metric space with a stratification satisfying the Frontier Condition and let $K \subseteq X$ be a closed union of strata.

(1) If $q: \mathrm{P}_{\mathrm{nsp}}(X, K) \rightarrow K$ is a stratified approximate fibration, then it is a stratified fibration.

(2) If $q: \operatorname{holink}_{\mathrm{s}}(X, K) \rightarrow K$ is a stratified approximate fibration, then it is a stratified fibration.

Proof. The proofs are similar so we only give the proof of (i). Suppose we are given 
Figure 8.5.3

a stratified lifting problem

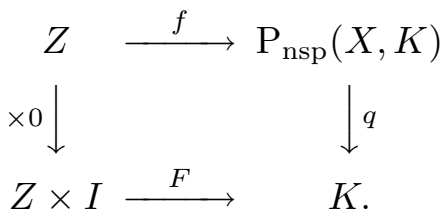

Thus, $F$ is a stratum preserving homotopy and the diagram commutes. According to Remark 5.5(2), we may assume that $Z$ is metric. Let $\tilde{F}: Z \times I \times[0,1) \rightarrow$ $\mathrm{P}_{\mathrm{nsp}}(X, K)$ be a stratified controlled solution so that $\tilde{F}$ is stratum preserving along $I \times[0,1), \tilde{F}(z, 0, t)=f(z)$ for all $(z, t) \in Z \times[0,1)$ and $\bar{F}: Z \times I \times I \rightarrow K$ defined by $\bar{F} \mid Z \times I \times[0,1)=q \tilde{F}$ and $\bar{F} \mid Z \times I \times\{1\}=F \times \operatorname{id}_{\{1\}}$ is continuous and stratum preserving along $I \times I$. Define $\hat{F}: Z \times I \rightarrow X^{I}$ by

$$
\hat{F}(z, y)(t)= \begin{cases}\tilde{F}(z, y, 1-t)(t), & \text { if } t>0 \\ F(z, y), & \text { if } t=0 .\end{cases}
$$

Note that $\hat{F}$ is continuous, $q \hat{F}=F$ and $\hat{F}(z, 0)=f(z)$. One checks that $\operatorname{Im} \hat{F} \subseteq$ $\mathrm{P}_{\text {nsp }}(X, K)$ and that $\hat{F}$ is a stratum preserving homotopy, so that $\hat{F}$ is a stratified solution to the given problem.

Lemma 8.7. Let $X$ be a metric space with a stratification satisfying the Frontier Condition and let $Y \subseteq X$ be a minimal stratum. If evaluation $q: \operatorname{holink}_{\mathrm{s}}(X, Y) \rightarrow$ $Y$ is a stratified fibration, then so is evaluation $q: \mathrm{P}_{\mathrm{nsp}}(X, Y) \rightarrow Y$.

Proof. Suppose we are given a stratified lifting problem

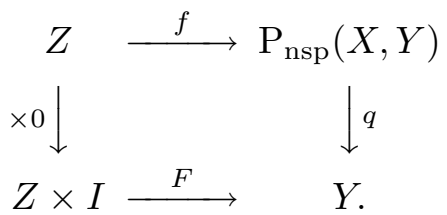


Let $A=\left\{z \in Z \mid f(z) \in Y^{I}\right\}$ and let $Z_{1}=Z \backslash A$. Thus, we have a stratified lifting problem

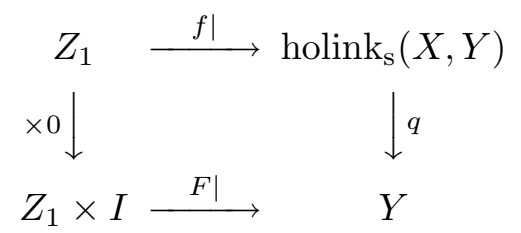

which by hypothesis has a stratified solution $G: Z_{1} \times I \rightarrow \operatorname{holink}_{\mathrm{s}}(X, Y)$. There is also a homotopy lifting problem

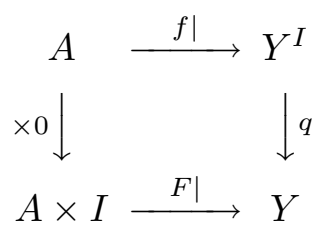

which has a lift $h: A \times I \rightarrow Y^{I}$ defined by

$$
h(z, s)(t)= \begin{cases}F(z, s(1-2 t))(t), & \text { if } 0 \leq t \leq 1 / 2 \\ f(z)(2 t-1), & \text { if } 1 / 2 \leq t \leq 1\end{cases}
$$

Note that $q h=F \mid A \times I$ but $h_{0} \neq f(z)$, so this is not a solution of the problem. At any rate we will now modify $G$ so that it can be extended to all of $Z \times I$ via $h$, and then worry about the initial lift.

Use Remark 5.5(2) to assume that $Z$ has a metric $d$ and use paracompactness to construct a map $u: Z_{1} \rightarrow(0,1]$ such that for each $(z, s) \in Z_{1} \times I$

$$
\operatorname{diam}\{G(z, s)(t) \mid 0 \leq t \leq u(z)\}<\operatorname{lub}\{d(f(z)(t), Y) \mid i \in I\}
$$

Note that $u$ extends to a map $Z \rightarrow I$ by sending all of $A$ to 0 . For each $z \in Z_{1}$ let $\gamma_{z}: I \rightarrow I$ be the map which takes $[0,1 / 2]$ linearly onto $[0, u(z)]$ and takes $[1 / 2,1]$ linearly onto $[u(z), 1]$. That is,

$$
\gamma_{z}(t)= \begin{cases}2 u(z) t, & \text { if } 0 \leq t \leq 1 / 2 \\ 2(t-1)(1-u(z))+1, & \text { if } 1 / 2 \leq t \leq 1\end{cases}
$$

Define $G^{\prime}: Z_{1} \times I \rightarrow \operatorname{holink}_{\mathrm{s}}(X, Y)$ by

$$
G^{\prime}(z, s)(t)= \begin{cases}G(z, s(1-2 t))\left(\gamma_{z}(t)\right), & \text { if } 0 \leq t \leq 1 / 2 \\ f(z)\left(\gamma_{z}(t)\right), & \text { if } 1 / 2 \leq t \leq 1\end{cases}
$$

Then there is a map $\tilde{G}: Z \times I \rightarrow \mathrm{P}_{\mathrm{nsp}}(X, Y)$ defined by $\tilde{G} \mid Z_{1} \times I=G^{\prime}$ and $\tilde{G} \mid A \times I=h$. Note that $q \tilde{G}=F$, but $\tilde{G}_{0} \neq f$. However, it is easy to see that there is a stratum preserving homotopy $H: f \simeq \tilde{G}_{0}$ such that $q H$ is the constant homotopy $F \times \mathrm{id}_{I}$. This is enough to conclude that $q: \mathrm{P}_{\mathrm{nsp}}(X, Y) \rightarrow Y$ is a stratified approximate fibration (cf. Lemma 5.6). Finally, apply Proposition 8.6 to conclude that $q$ is a stratified fibration. 


\section{Proofs of the MAin Results}

In this section the proofs of the main results stated in $\S 6$ are presented. The first result is a restatement of Theorem 6.7(1). A couple of related results are given in Corollaries 9.5 and 9.7.

Proposition 9.1. For $k \geq 0, l \geq 2$, Statements $T_{l-1,1}, T_{k, 1}, T_{k, l-1}$ and $S_{k, l-1}$ imply Statement $T_{k, l}$.

Proof. Let $Y$ be a closed union of strata of a homotopically stratified metric space $X$ such that $X \backslash Y$ has $k$ strata and $Y$ has $l$ strata. Let $Y_{0}$ be a minimal stratum of $Y$ and note that

(1) $Y_{0}$ is stratified forward tame in $Y$ by $T_{l-1,1}$,

(2) $Y \backslash Y_{0}$ is stratified forward tame in $X \backslash Y_{0}$ by $T_{k, l-1}$,

(3) $Y_{0}$ is stratified forward tame in $(X \backslash Y) \cup Y_{0}$ by $T_{k, 1}$, and

(4) $\mathrm{P}_{\mathrm{nsp}}\left(X \backslash Y_{0}, Y \backslash Y_{0}\right) \rightarrow Y \backslash Y_{0}$ is a stratified fibration by $S_{k, l-1}$.

The Blending Proposition 8.5 (applied with $K=Y$ and $L=M=Y_{0}$ ) implies that $Y$ is stratified forward tame in $X$.

The following result is a restatement of Theorem 6.7(2).

Proposition 9.2. For $k \geq 2$ Statements $T_{k-1,1}$ and $S_{k-1,1}$ imply Statement $S_{k, 1}$.

Proof. Let $Y$ be a closed union of strata of a homotopically stratified metric space $X$ such that $Y$ is a single stratum and $X \backslash Y$ has $k$ strata. We need to show that $q: \mathrm{P}_{\text {nsp }}(X, Y) \rightarrow Y$ is a stratified fibration. By Lemma 8.7 it suffices to show that $q: \operatorname{holink}_{\mathrm{s}}(X, Y) \rightarrow Y$ is a stratified fibration.

Let $X_{0}$ be a minimal stratum of $X \backslash Y$ and note that $W=X_{0} \cup Y$ is a closed union of strata of $X$ with only two strata such that $X \backslash W$ has $k-1$ strata. Thus $T_{k-1,1}$ implies that $X_{0}$ is stratified forward tame in $X \backslash Y$. Lemma 8.4 implies that there exists an open neighborhood $U^{\prime}$ of $W \backslash Y=X_{0}$ in $X \backslash Y$ such that $W$ is stratified forward tame in $U^{\prime} \cup Y$. Let $U$ be an open neighborhood of $W$ in $U^{\prime} \cup Y$ and $h: U \times I \rightarrow U^{\prime} \cup Y$ a nearly stratum preserving deformation of $U$ to $W$ in $U^{\prime} \cup Y$ as in Definition 3.4. Moreover, the proof of Lemma 8.4 shows that we may assume that $h^{-1}(Y)=Y \times I$.

Observe that $q: \mathrm{P}_{\mathrm{nsp}}\left(X \backslash X_{0}, Y\right) \rightarrow Y$ is a stratified fibration by $S_{k-1,1}$. As observed in the proof of Corollary 6.2(2), it follows from Remark 5.5(1) that $q$ : $\operatorname{holink}_{\mathrm{s}}\left(X \backslash X_{0}, Y\right) \rightarrow Y$ is a stratified fibration.

We will now show that $q: \operatorname{holink}_{\mathrm{s}}(U, Y) \rightarrow Y$ is a stratified fibration. Suppose there is given a stratified lifting problem

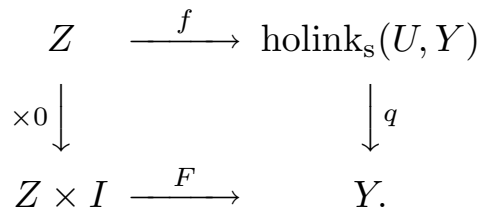

Note that the adjoint induces $\hat{h}_{1}: \operatorname{holink}_{\mathrm{s}}(U, Y) \rightarrow \operatorname{holink}_{\mathrm{s}}(W, Y)$ so that we have a stratum preserving lifting problem

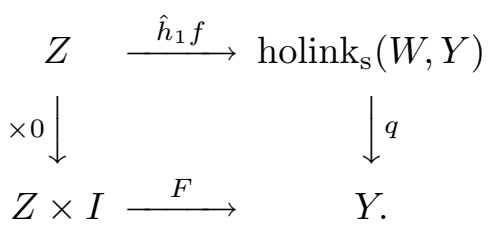


Now $S_{1,1}$ (which holds according to Proposition 6.6) implies that there is a stratified solution $G: Z \times I \rightarrow \operatorname{holink}_{\mathrm{s}}(W, Y)$ of this second problem (as above, we are using the fact that $\mathrm{P}_{\mathrm{nsp}}(W, Y) \rightarrow Y$ is a fibration implies that holink $\mathrm{s}_{\mathrm{s}}(W, Y) \rightarrow Y$ is a stratified fibration). Define $\hat{G}: Z \times I \times I \rightarrow W$ by $\hat{G}(z, s, t)=G(z, s)(t)$ and $g: Z \times I \rightarrow \mathrm{P}_{\mathrm{nsp}}(X, W)$ by $g(z, s)(t)=h(f(z)(s), 1-t)$ so that there is a stratified lifting problem

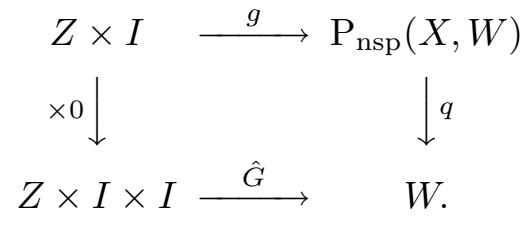

Unfortunately, it takes $S_{k-1,2}$ to solve this problem. So instead of attempting to solve it, note that it restricts to

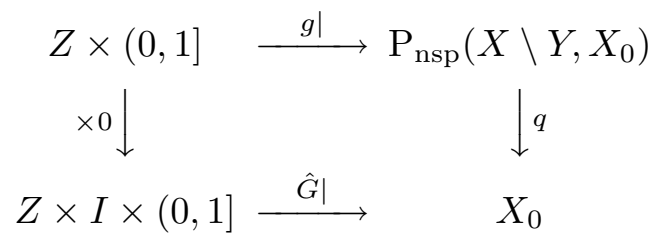

which, by $S_{k-2,1}$, has a stratified solution $G^{*}: Z \times I \times(0,1] \rightarrow \mathrm{P}_{\mathrm{nsp}}\left(X \backslash Y, X_{0}\right)$. We will now define a commuting diagram

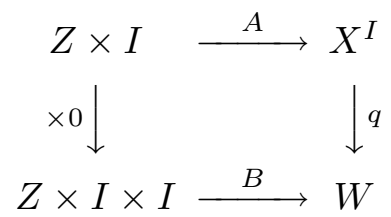

to which the Limbo Lemma 8.1 can be applied. Define $B$ and $A$ by the formulas $B(z, r, s)=\hat{G}(z, r, 1-s)=G(z, r)(1-s)$ and $A(z, r)=G^{*}(z, r, 1)$. One checks that the diagram commutes. Define $C: Z \times I \times[0,1) \rightarrow X^{I}$ by $C(z, r, s)=G^{*}(z, r, 1-s)$. Then $q C=B \mid$ and $C(z, r, 0)=A(z, r)$ so that $C$ is a partial lift in the sense of 8.1. It follows from 8.1 that there exists a lift $D: Z \times I \times I \rightarrow X^{I}$ such that $q D=B$ and $D(z, r, 0)=A(z, r)$. Now define $E: Z \times I \rightarrow X^{I}$ by $E(z, r)=D(z, r, 1)$. Then

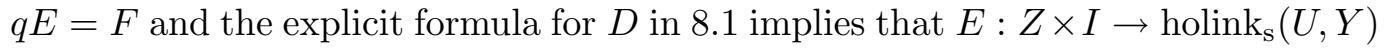
and that there is a stratum preserving homotopy $E_{0} \simeq f$ which is fibre preserving over $Y$. This is enough to conclude that there exists a stratified controlled solution of (9.2.1) (cf. 5.6 and $[10, \S 12])$. Hence, $q: \operatorname{holink}_{\mathrm{s}}(U, Y) \rightarrow Y$ is a stratified approximate fibration and Proposition 8.6 implies that it is a stratified fibration.

We now complete the proof that $q: \operatorname{holink}_{\mathrm{s}}(X, Y) \rightarrow Y$ is a stratified fibration. Consider a stratified lifting problem

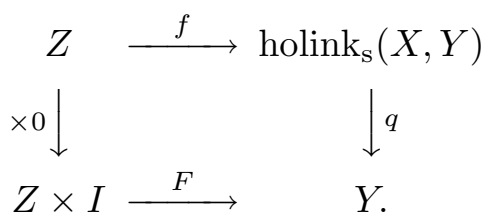

Assume that $Z$ is metric $(5.5(2))$. Let

$$
\begin{aligned}
& Z_{0}=\left\{z \in Z \mid f(z) \in \operatorname{holink}_{\mathrm{s}}(W, Y)\right\}, \text { and } \\
& Z_{1}=\{z \in Z \mid f(z) \cap(X \backslash U) \neq \emptyset\} .
\end{aligned}
$$


Then problem (9.2.2) restricts to problems

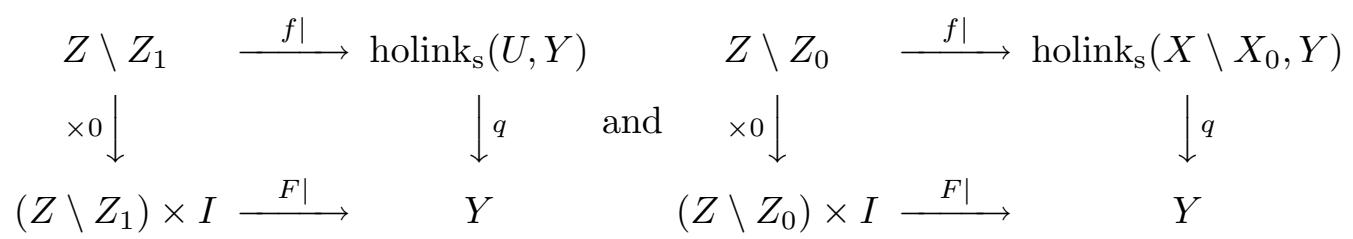

which (by the first two parts of this proof) have stratified solutions $G:\left(Z \backslash Z_{1}\right) \times$ $I \rightarrow \operatorname{holink}_{\mathrm{s}}(U, Y)$ and $H:\left(Z \backslash Z_{0}\right) \times I \rightarrow \operatorname{holink}_{\mathrm{s}}\left(X \backslash X_{0}, Y\right)$. It follows that $G|, H|:\left(Z \backslash\left(Z_{0} \cup Z_{1}\right)\right) \times I \rightarrow \operatorname{holink}_{\mathrm{s}}\left(X \backslash X_{0}, Y\right)$ are both stratified solutions of

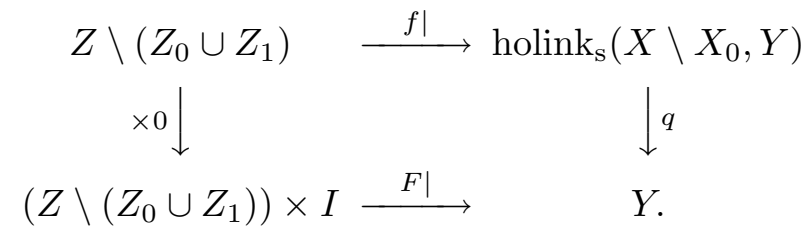

By Lemma 5.3 there exists a map $J: Z \backslash\left(Z_{0} \cup Z_{1}\right) \times I \times I \rightarrow \operatorname{holink}_{\mathrm{s}}\left(X \backslash X_{0}, Y\right)$ such that

(1) $q J=F \mid \times \operatorname{id}_{I}$,

(2) $J$ is stratum preserving along $I \times I$,

(3) $J(z, 0, t)=f(z)$ for each $(z, t) \in Z \backslash\left(Z_{0} \cup Z_{1}\right) \times I$,

(4) $J(z, s, 0)=G(z, s)$ and $J(z, s, 1)=H(z, s)$ for each $(z, s) \in Z \backslash\left(Z_{0} \cup Z_{1}\right) \times I$.

Let $\varphi: Z \rightarrow I$ be a map such that $\varphi^{-1}(0)=Z_{0}$ and $\varphi^{-1}(1)=Z_{1}$. Finally, define $\tilde{F}: Z \times I \rightarrow \operatorname{holink}_{\mathrm{s}}(X, Y)$ by

$$
\tilde{F}(z, t)= \begin{cases}G(z, t), & \text { if } z \in Z_{0} \\ J(z, t, \varphi(z)), & \text { if } z \in Z \backslash\left(Z_{0} \cup Z_{1}\right) \\ H(z, t), & \text { if } z \in Z_{1} .\end{cases}
$$

It follows that $\tilde{F}$ is a stratified solution of $(9.2 .2)$.

The following result is a restatement of Theorem 6.7(3).

Proposition 9.3. For $k \geq 2$ Statements $T_{k-1,1}$ and $S_{k-1,1}$ imply Statement $T_{k, 1}$.

Proof. Let $Y$ be a closed union of strata of a homotopically stratified metric space $X$ such that $Y$ is a single stratum and $X \backslash Y$ has $k$ strata. Let $X_{0}$ be a minimal stratum of $X \backslash Y$ and note that $W=X_{0} \cup Y$ is a closed union of strata of $X$ with only two strata such that $X \backslash W$ has $k-1$ strata. Note that

(1) $Y$ is stratified forward tame in $W$ by $T_{1,1}$ (see $6.5(3)$ ),

(2) $X_{0}$ is stratified forward tame in $X \backslash Y$ by $T_{k-1,1}$,

(3) $Y$ is stratified forward tame in $X \backslash X_{0}$ by $T_{k-1,1}$, and

(4) $\mathrm{P}_{\mathrm{nsp}}\left(X \backslash Y, X_{0}\right) \rightarrow X_{0}$ is a stratified fibration by $S_{k-1,1}$.

The Blending Proposition 8.5 (applied with $K=W$ and $L=M=Y_{0}$ ) implies that $Y$ is stratified forward tame in $X$.

The following result is a restatement of Theorem $6.7(4)$. 
Proposition 9.4. For $k \geq 0, l \geq 0$, Statements $T_{k+l, 1}, S_{k+l, 1}$ and $S_{k, l}$ imply Statement $S_{k, l+1}$.

Proof. Let $Y$ be a closed union of strata of a homotopically stratified space metric $X$ such that $X \backslash Y$ has $k$ strata and $Y$ has $l+1$ strata, and consider the map $q: \mathrm{P}_{\mathrm{nsp}}(X, Y) \rightarrow Y$. Let $Y_{0}$ be a minimal stratum of $Y$. Note that $q^{-1}\left(Y_{0}\right)=$ $\mathrm{P}_{\text {nsp }}\left(X, Y_{0}\right)$ and $q^{-1}\left(Y \backslash Y_{0}\right)=\mathrm{P}_{\text {nsp }}\left(X \backslash Y_{0}, Y \backslash Y_{0}\right)$. Since $\left(X \backslash Y_{0}\right) \backslash\left(Y \backslash Y_{0}\right)=X \backslash Y$, it follows that $\left(X \backslash Y_{0}\right) \backslash\left(Y \backslash Y_{0}\right)$ has $k$ strata. Also, $Y \backslash Y_{0}$ has $l$ strata. From $S_{k, l}$ we have that $q \mid: q^{-1}\left(Y \backslash Y_{0}\right) \rightarrow Y \backslash Y_{0}$ is a stratified fibration. Since $X \backslash Y_{0}$ has $k+l$ strata and $Y_{0}$ has 1 stratum, $S_{k+l, 1}$ implies that $q \mid: q^{-1}\left(Y_{0}\right) \rightarrow Y_{0}$ is a stratified fibration. Moreover, $T_{k+l, 1}$ implies that $Y_{0}$ is stratified forward tame in $X$. It follows from Corollary 7.3 that $Y_{0}$ is a $q$-NDR subset of $Y$. Now Lemma 7.5 implies that $q: \mathrm{P}_{\mathrm{nsp}}(X, Y) \rightarrow Y$ is a stratified approximate fibration. Finally, use Proposition 8.6 to conclude that $q$ is a stratified fibration.

Finally we establish a couple of related results.

Corollary 9.5. Let $X$ be a homotopically stratified metric space with a finite number of strata and let $Y \subseteq X$ be a closed union of strata. Then $q: \mathrm{P}_{\mathrm{nsp}}(X, Y) \rightarrow Y$ is a stratified system of stratified fibrations.

Proof. If $Y^{k}$ is a skeleton of $Y$ (i.e., $Y^{k}=X^{k} \cap Y$ ), then Corollary 7.3 implies that $Y^{k}$ is a $q$-NDR subset of $Y$. Since $q^{-1}\left(Y_{k}\right)=\mathrm{P}_{\mathrm{nsp}}\left(X, Y_{k}\right)$, Corollary 6.2(4) implies that $q \mid: q^{-1}\left(Y_{k}\right) \rightarrow Y_{k}$ is a stratified fibration.

Lemma 9.6. Let $X$ be a homotopically stratified metric space with a finite number of strata and let $Y \subseteq X$ be a minimal stratum. Then $q: \operatorname{holink}(X, Y) \rightarrow Y$ is a fibration.

Proof. The proof of Proposition 8.6 shows that it suffices to show that

$$
q: \operatorname{holink}(X, Y) \rightarrow Y
$$

is an approximate fibration (cf. [14, 2.7]). Let

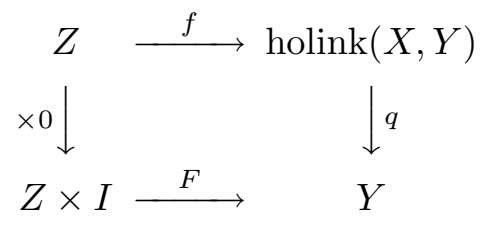

be a lifting problem. By Theorem $6.3 Y$ is stratified forward tame in $X$. Thus let $U$ be an open neighborhood of $Y$ in $X$ for which there is a nearly stratum preserving homotopy $h: U \times I \rightarrow X$ as in Definition 3.4. By using an elementary partition of unity argument, one sees that we may assume that $f(Z) \subseteq \operatorname{holink}(U, Y)$ (cf. [2], $[14,2.4(1)])$. Now define $f^{\prime}: Z \times I \rightarrow X^{I}$ by

$$
f^{\prime}(z, s)(t)=h(f(z)(s), 1-t) .
$$

Since $h$ is nearly stratum preserving, $f^{\prime}(Z \times(0,1]) \subseteq \operatorname{holink}_{\mathrm{s}}(X, Y)$. Moreover, $f^{\prime}(z, 0)$ is the constant path at $f(z)(0)$. Thus we have $f^{\prime}: Z \times I \rightarrow \mathrm{P}_{\mathrm{nsp}}(X, Y)$. Define $F^{\prime}: Z \times I \times I \rightarrow Y$ by

$$
F^{\prime}(z, s, t)= \begin{cases}(h(f(z)(s-t), 1), & \text { if } 0 \leq t \leq s \\ F(z, t-s), & \text { if } s \leq t \leq 1\end{cases}
$$


and note that we have a stratified lifting problem

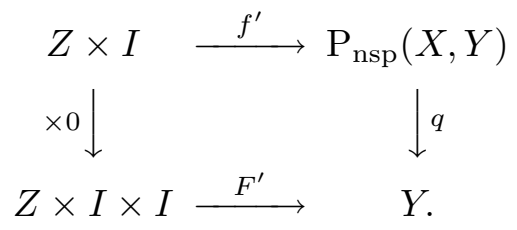

By Theorem 6.1 this problem has a stratified solution $\tilde{F}^{\prime}: Z \times I \times I \rightarrow \mathrm{P}_{\mathrm{nsp}}(X, Y)$. Define $g: Z \times I \times I \rightarrow \mathrm{P}_{\mathrm{nsp}}(X, Y)$ by

$$
g(z, s, t)(u)=\tilde{F}^{\prime}(z, u, s)(1-t+t u) .
$$

One checks that

(1) $g(z, 0,0)=f(z)$,

(2) $g(z, s, 1)(0)=F(z, s)$, and

(3) $g$ is stratum preserving along $I \times I$.

We now modify $g$ to get a controlled stratified solution $\tilde{F}$ to the original problem (9.6.1). To this end define $\tilde{F}: Z \times I \times[0,1) \rightarrow \mathrm{P}_{\text {nsp }}(X, Y)$ by $\tilde{F}(z, s, t)(u)=$ $g(z s w)(u)$ where

$$
w= \begin{cases}\frac{t s}{1-t} & \text { if } 0 \leq s \leq \frac{1-t}{2-t} \\ \frac{t}{2-t}, & \text { if } \frac{1-t}{2-t} \leq s \leq 1 .\end{cases}
$$

Then $\tilde{F}(z, 0, t)=g(z, 0,0)=f(z), \tilde{F}$ is stratum preserving along $I \times[0,1)$, and $w \rightarrow 1$ as $t \rightarrow 1$ so that $q \tilde{F}$ extends continuously to $Z \times I \times I$ via $F \times \operatorname{id}_{\{1\}}$.

The following result is essentially due to Quinn [14]. See $\S 10$.

Corollary 9.7. Let $X$ be a homotopically stratified metric space with a finite number of strata and let $Y \subseteq X$ be a closed union of strata. Then $q: \operatorname{holink}(X, Y) \rightarrow Y$ is a stratified fibration and a stratified system of fibrations. (Here holink $(X, Y)$ is unstratified.)

Proof. It suffices to show that $q: \operatorname{holink}(X, Y) \rightarrow Y$ is a stratified system of fibrations, for then Corollary 7.6 implies that $q$ is a stratified approximate fibration and the proof of Proposition 8.6 shows that $q$ is also a stratified fibration. Since a stratum $Y_{j}$ is minimal in $X \backslash \bigcup_{i<j} Y_{i}$ and $q^{-1}\left(Y_{j}\right)=\operatorname{holink}\left(X \backslash \bigcup_{i<j} Y_{i}, Y_{j}\right)$, Lemma 9.6 implies that $q \mid: q^{-1}\left(Y_{j}\right) \rightarrow Y_{j}$ is a fibration. It remains to see that the skeleton $Y^{j}$ is a $q$-NDR subset of $Y$. Using the fact that $Y^{j}$ is stratified forward tame in $Y$ (Theorem 6.3), this follows from the proof of Lemma 7.2.

\section{Appendix: Pure subsets}

Versions of Theorem 6.3 and Corollary 9.7 are claimed by Quinn in [14, 3.2] for subsets more general than closed unions of strata, namely the so-called pure subsets. In this section we present an example to show that $[14,3.2]$ is not quite true in the generality as stated, and then show, if one assumes the strata are locally path connected, Quinn's claim can be recovered from the results in this paper. Of course, local path connectedness is not a burdensome restriction because in Quinn's important applications the strata are manifolds. 
Definition 10.1. A subset $A$ of a space $X$ with a stratification is called a pure subset if $A$ is closed and a union of components of strata of $X$.

Let $Y=\{0,1 / n \mid n=1,2,3, \ldots\} \subseteq \mathbb{R}$ and let $X$ be the cone on $Y$ with vertex $v \in X$. Then $X$ has a natural stratification with two strata: $\{v\}$ and $X \backslash\{v\}$. It is easy to see that with this stratification, $X$ is a homotopically stratified metric space. The subset $A \subseteq X$ consisting of the closed segment joining $\{0\}$ and $\{v\}$ is a pure subset of $X$, but $A$ is not stratified forward tame in $X$ (or even a neighborhood deformation retract). This contradicts [14, 3.2].

The next result shows that in some situations spaces with stratifications can be restratified so that a pure subset becomes a closed union of strata, rather than just a closed union of components of strata, so that the results of this paper apply.

For notation in Proposition 10.2 let $X$ denote a space with a finite filtration by closed subsets:

$$
\emptyset=X^{-1} \subseteq X^{0} \subseteq X^{1} \subseteq \cdots \subseteq X^{n}=X .
$$

Assume that the strata $X_{i}=X^{i} \backslash X^{i-1}$ satisfy the Forward Tameness and Normal Fibrations Conditions of Definition 3.3. This might be slightly confusing because we are not now assuming that the Frontier Condition holds. However, the following weaker version of the Frontier Condition is satisfied: If $C$ is a path component of a stratum $X_{i}$ and $C \cap \operatorname{cl}\left(X_{j}\right) \neq \emptyset$ for some stratum $X_{j}$, then $C \subseteq \operatorname{cl}\left(X_{j}\right)$. In fact there is a component $K$ of $X_{j}$ such that $C \subseteq \operatorname{cl}(K)$. For if $C \cap \operatorname{cl}\left(X_{j}\right) \neq \emptyset$, then Forward Tameness guarantees that holink $\left(X_{j} \cup C, C\right) \neq \emptyset$ and the path connectivity of $C$ and the Normal Fibrations condition imply the existence of $K$.

Proposition 10.2. Let $X$ be as above. Suppose that the strata are locally path connected and let $A \subseteq X$ be a pure subset. Then there exists a stratification $\mathcal{R}$ of $X$ such that:

(1) $\mathcal{R}$ is finite, satisfies the Frontier Condition, and elements of $\mathcal{R}$ are locally closed,

(2) each $R \in \mathcal{R}$ is a union of components of the strata $\left\{X_{i}\right\}$,

(3) $X$ is homotopically stratified with respect to $\mathcal{R}$, and

(4) $A$ is a closed union of some of the strata $\mathcal{R}$.

Proof. The proof is by induction on $n$. If $n=0$, then $A$ is closed and a union of components of $X=X_{0}$. Since $X$ is locally path connected, $A$ is also open. Let $\mathcal{R}=\{X \backslash A, A\}$. Clearly $\mathcal{R}$ is finite and each member of $\mathcal{R}$ is a union of components of $X$. Because the strata are both open and closed, the Frontier, Forward Tameness and Normal Fibrations Conditions trivially hold.

Now assume that $n>0$ and that the result is true for filtrations with fewer than $(n+1)$-skeleta. In particular, $A \backslash X_{0}$ is pure in $X \backslash X_{0}$ and the result applies to

$$
\emptyset=X^{0} \backslash X_{0} \subseteq X^{1} \backslash X_{0} \subseteq \cdots \subseteq X^{n} \backslash X_{0}=X \backslash X_{0} .
$$

Let $\mathcal{S}$ be the stratification of $X \backslash X_{0}$ with the guaranteed properties. Let $\left\{C_{\alpha}\right\}$ be the collection of components of $X_{0}$. Define $C_{\alpha} \sim C_{\beta}$ to mean for every $S \in \mathcal{S}$, $C_{\alpha} \cap \operatorname{cl}(S) \neq \emptyset$ if and only if $C_{\beta} \cap \operatorname{cl}(S) \neq \emptyset$. For $C_{\alpha} \subseteq X \backslash Y$ let

$$
\left[C_{\alpha}\right]_{1}=\cup_{\beta}\left\{C_{\beta} \mid C_{\beta} \subseteq X \backslash Y \text { and } C_{\alpha} \sim C_{\beta}\right\}
$$

and for $C_{\alpha} \subseteq Y$ let

$$
\left[C_{\alpha}\right]_{2}=\cup_{\beta}\left\{C_{\beta} \mid C_{\beta} \subseteq Y \text { and } C_{\alpha} \sim C_{\beta}\right\}
$$


Let $\mathcal{R}=\mathcal{S} \cup\left\{\left[C_{\alpha}\right]_{1}\right\} \cup\left\{\left[C_{\alpha}\right]_{2}\right\}$. That $\mathcal{R}$ is finite follows from the fact that $\mathcal{S}$ is finite and there exist natural injections of $\left\{\left[C_{\alpha}\right]_{k}\right\}_{\alpha}$ into the set of subsets of $\mathcal{S}$ for $k=1,2$. We only need to check forward tameness and normal fibrations at the new strata $\mathcal{R} \backslash \mathcal{S}$. The union of these strata is $X_{0}$ and their components are both closed and open in $X_{0}$. The result now follows from the fact that the original stratification satisfies forward tameness and normal fibrations at $X_{0}$. The Frontier Condition is verified as follows. Let $R \in \mathcal{R} \backslash \mathcal{S}$ and suppose $R \cap \operatorname{cl}(S) \neq \emptyset$ for some $S \in \mathcal{S}$. This implies that $C_{\alpha} \cap \operatorname{cl}(S) \neq \emptyset$ whenever $R=\left[C_{\alpha}\right]_{k}$. To show $R \subseteq \operatorname{cl}(S)$ it suffices to show that $C_{\alpha} \subseteq \operatorname{cl}(S)$. Since $S=\cup_{i=1}^{n} S \cap X_{i}$ there exists $i=1, \ldots, n$ such that $C_{\alpha} \cap \operatorname{cl}\left(S \cap X_{i}\right) \neq \emptyset$. By the comments before the statement of 10.2, there exists a component $K$ of $X_{i}$ such that $C_{\alpha} \subseteq \operatorname{cl}(K)$. Since $S$ is a union of components of strata from the original stratification, $K \subseteq S$ and so $C_{\alpha} \subseteq \operatorname{cl}(S)$.

Elements of $\mathcal{R}$ are easily seen to be locally closed. Finally note that $A=$ $\cup_{\alpha}\left\{\left[C_{\alpha}\right]_{2}\right\}$.

In the proof above one cannot simply let $\mathcal{R}$ be the collection of components of the strata $\left\{X_{i}\right\}$ because the components need not be locally finite. For example, let $X$ be the space consisting of a point $\{v\}$ with a countable collection of closed line segments emanating from and converging to $\{v\}$. Stratify $X$ with two strata: $\{v\}$ and $X \backslash\{v\}$. The the collection of components of strata is not locally finite.

Restratifications also appear in the work of Beshears [2].

Proposition 10.2 can be used to recover Quinn's result [14, 3.2] from Theorem 6.3 and Corollary 9.7 if one assumes strata are locally path connected.

\section{REFERENCES}

1. S. Akbulut and H. King, Topology of real algebraic sets, Math. Sci. Research Inst. Publ. 25, Springer-Verlag, New York, 1992.

2. A. Beshears, G-isovariant structure sets and stratified structure sets, Vanderbilt Univ. preprint, 1997.

3. S. Cappell and J. Shaneson, The mapping cone and cylinder of a stratified map, Prospects in topology; Proceedings of a conference in honor of William Browder (F. Quinn, ed.), Ann. of Math. Studies, vol. 138, Princeton Univ. Press, Princeton, 1995, pp. 58-66.

4. K. H. Dovermann and R. Schultz, Equivariant surgery theories and their periodicity properties, Lecture Notes in Math. 1443, Springer-Verlag, New York, 1990.

5. M. Goresky and R. MacPherson, Stratified Morse theory, Ergeb. Math. Grenzgeb (3) 14, Springer-Verlag, New York, 1988.

6. B. Hughes, Geometric topology of stratified spaces, Electron. Res. Announc. Amer. Math. Soc. 2 (1996), 73-81.

7. The geometric topology of stratified spaces (in preparation).

8. B. Hughes and A. Ranicki, Ends of complexes, Cambridge Tracts in Math. 123, Cambridge Univ. Press, Cambridge, 1996.

9. B. Hughes, L. Taylor, S. Weinberger and B. Williams, Neighborhoods in stratified spaces with two strata (preprint).

10. B. Hughes, L. Taylor and B. Williams, Bundle theories for topological manifolds, Trans. Amer Math. Soc. 319 (1990), 1-65.

11. J. Mather, Notes on topological stability, Harvard Univ., Cambridge, 1970 (photocopied).

12. __ Stratifications and mappings, Dynamical Systems (M. M. Peixoto, ed.), Proc. Sympos., Univ. Bahia, Salvador, Brazil, 1971, Academic Press, New York, 1973, pp. 195-232.

13. F. Quinn, Ends of maps. II, Invent. Math. 68 (1982), 353-424.

14. _ Homotopically stratified sets, J. Amer. Math. Soc. 1 (1988), 441-499.

15. E. Spanier, Algebraic topology, McGraw-Hill, New York, 1966.

16. R. Talbert, Stratified and equivariant homology via homotopy colimits, Ph.D. thesis, Vanderbilt Univ., 1997. 
17. A. Verona, Stratified mappings - structure and triangulability, Lecture Notes in Math. 1102, Springer-Verlag, New York, 1984.

18. G. W. Whitehead, Elements of homotopy theory, Graduate Texts in Math. 61, SpringerVerlag, New York, 1978.

Department of Mathematics, Vanderbilt University, Nashville, Tennessee 37240

E-mail address: hughes@math.vanderbilt.edu 